\title{
Full Range Swept-Source Optical Coherence Tomography with Ultra Small Fiber Probes for Biomedical Imaging
}

\author{
Youxin Mao, Costel Flueraru and Shoude Chang \\ Institute for Microstructural Sciences, National Research Council Canada \\ 1200 Montreal Rd, Ottawa, K1A 0R6, ON, Canada
}

\section{Introduction}

Optical coherence tomography (OCT) (Huang et al., 1991) is becoming an increasingly important imaging tool for many applications in biology and medicine, such as diagnosis and guided surgery. Due to its high resolution and fiber catheter capability, OCT is more attractive than current imaging technologies, such as ultrasound. An OCT system with higher sensitivity is essentially important for imaging the biomedical turbid tissue because the backscattered optical signal from the tissue is extremely weak. In the earlier stages of OCT imaging, axial (depth) ranging is provided by linearly scanned low-coherence interferometry (Youngquist et al., 1987; Takada et al., 1987). This method of OCT, referred to as time-domain OCT (TD-OCT), has a relatively slow sensitivity and imaging speed because its sensitivity is inversely proportional to the imaging speed. Fourier domain techniques in OCT have received much attention in recent years due to its significant sensitivity and speed advantages over TD-OCT (Leitgeb et al., 2003; aChoma et al., 2003; De Boer et al., 2003). Fourier domain methods include spectral-domain OCT (SD-OCT) and swept-source OCT (SS-OCT). In SD-OCT, individual spectral components of low coherence light are detected separately by the use of a spectrometer and a charge-coupled device (CCD) array (Fercher et al., 1995; Hausler \& Lindner, 1998) CCD arrays however may introduce phase washout problems during the pixel integration time. Furthermore, detection using a spectrometer and CCD array cannot implement differential optical detection. SS-OCT uses a wavelengthswept laser source and photodetectors based on optical frequency-domain reflectometry for imaging (Chinn et al., 1997; aYun et al., 2003). SS-OCT is particularly important for imaging in the $1.3 \mu \mathrm{m}$ wavelength range, where low-cost detector arrays are not available. The larger penetration depth of the OCT image by using the $1.3 \mu \mathrm{m}$ wavelength light source is important for the biomedical turbid tissues, such as human skin and arterial plaque, in comparison to that by using $1.0 \mu \mathrm{m}$ or shorter wavelength light source. SS-OCT could also make possible for a quadrature interferometry based on multi-port fiber couplers, for example, $3 \times 3$ quadrature interferometer (bChoma et al., 2003; aMao et al., 2008). Due to its ability to have instantaneous complex signals with stable phase information, OCT with a $3 \times 3$ quadrature interferometer could suppress the complex conjugate artifact naturally, therefore 
to double the effective imaging depth. By detection of the phase from the complex signals, it also could exploit additional information of the tissue to enhance image contrast, obtain functional information, and perform quantitative measurements (Sticker et al., 2001; aZhao et la. 2000). In addition, SS-OCT could make possible for an unbalanced input fiber interferometer and differential output detection by using a Mach-Zehnder interferometer. The unbalanced input could emit larger portion of the optical power from optical source to the tissue than that to the reference mirror for increasing sensitivity (Rollins \& Izatt, 1999). The differential detection is used to reduce the excess intensity noise to further sensitivity enhancement compared to SD-OCT (Podoleanu, 2000).

In SS-OCT, the location of a scatterer within tissue is obtained by a Fourier transformation of the optical measurement. When the real component of the interferometric signal is the only detected part, a complex conjugate artifact is introduced after the Fourier transformation. This artifact prevents the distinction between positive and negative object depths thereby reducing the effective imaging range by half. As imaging range is important in biomedical applications, methods for removing this complex conjugate artifact to achieve full range in SS-OCT are of significant interest. Different full-range SS-OCT imaging methods which measure the complex component of the interferometric signal by shifting the phase of the reference and/or sample reflections have been reported. This phase shift has been implemented by a high-speed electronic-optical phase modulator (Zhang et al., 2004), two high-speed acoustic-optical frequency shifters (Yun et al., 2004), and a pair of linearly polarized beams (Vakoc et al., 2006). All of these methods suffered from significant image corruption resulting from any small variations in the phase shift or birefringence of used materials. Recently, acquisition of both real and imaginary interferometric components was demonstrated using Michelson quadrature interferometers using $3 \times 3$ fused fiber couplers and non-differential optical detection (Choma et al., 2003; Sarunic et al., 2005; Sarunic et al., 2006). In reference (Sarunic et al., 2005), a 3×3 Michelson quadrature interferometer with balanced differential detection was used for acquiring the complex interferometric signal. Signal attenuation was used to achieve such balanced differential detection which resulted in loss of optical power. In this system, there was a non-complementary phase shift of $60^{\circ}$ between the two output interferometric signals that needed to be converted to quadrature components by a trigonometric manipulation. In addition, due to the nature of the Michelson interferometer, the optical output power at one of the ports of the $3 \times 3$ coupler ( $1 / 3$ of the source power) was not utilized in these references.

A wavelength-swept laser source with high-speed, high power, long coherence, i.e. narrow instantaneous linewidth, and wide sweeping range is essential for SS-OCT because the imaging speed, sensitivity, depth and resolution directly rely on the sweeping rate, output power, coherence length and sweeping range of the source. A high powered wavelengthswept laser is also needed for multi-channel SS-OCT. Much progress has been made on the development of high-speed swept lasers, but their output power has been limited. A sweeping repetition rate of $115 \mathrm{kHz}$ has been demonstrated by using a 128-facet polygon scanner (Oh et al., 2005). Because the cavity length in this swept laser is not short enough, the photons do not have sufficient time within the laser cavity to build up the optical power and to narrow the linewidth through mode competition. That is why the resultant optical average power and instantaneous linewidth were low $(23 \mathrm{~mW})$ and wide $(0.23 \mathrm{~nm})$, 
respectively. A better result of higher average power of $54 \mathrm{~mW}$ (Oh et al., 2008) has been reported, but no description of the laser system has been given. Fourier-domain mode locking (FDML) technique is an alternative approach to achieving higher sweeping speed while a higher optical power is preserved, which was reported recently (Huber et al., 2006a). An FDML wavelength-swept laser with a long cavity has a quasi-stationary operation where one wavelength propagates through the cavity and returns to an optical narrow bandpass filter. Consequently, the laser generates a sequence of narrowband optical wavelength sweeps at the cavity repetition rate. An FDML wavelength swept laser with sweeping repetition up to $370 \mathrm{kHz}$ using a Fabry-Perot tunable filter has been reported (Huber et al., 2006b). Although a narrow $(\sim 0.1 \mathrm{~nm})$ instantaneous linewidth was reached, the direct output power of this laser was low. To achieve an average output power of $36 \mathrm{~mW}$ at the sweeping repetition of $100 \mathrm{kHz}$, an external semiconductor optical power booster had to be used. However, an amplifier outside the cavity could cause performance degradation, e.g., an increase in linewidth (Huber et al., 2005) and system noise (Rao et al., 2007), thereby reducing the penetration depth and sensitivity of an OCT system.

In the most optically nontransparent tissues, OCT has a typical imaging depth limitation of 1-3 mm. As a result, the earliest in vivo OCT imaging of tissue microstructure and microvasculature was restricted to a few transparent or superficial organ sites, such as the retina (Yazdanfar et al., 2000; White et al., 2003) and skin (Zhao et al., 2000). To overcome this depth limitation, optical probes, such as endoscopes, catheters, and needles have been investigated for in vivo OCT imaging in mucosal layers of the gastrointestinal tract (Tran et al., 2004; Yang et al., 2005a), deep organs and tissues (Li et al., 2000; Yang et al., 2005b), and inter-arterial and intra-vascular (Fujimoto et al., 1999; Diaz-Sandoval et al., 2005). However, for the imaging of small lumen, narrow space, and deep tissue and organ of humans and small animals, a key concern is the possible damage from the mechanical insertion of the optical probe. Therefore it is critical to develop an ultra-small optical probe that is compatible with the current optical biomedical imaging systems, which results in minimum tissue damage. In vivo optical imaging of internal tissues is generally performed using a fiber-optic probe, since an optical fiber can be easily and cheaply produced with a diameter of less than $150 \mu \mathrm{m}$. The key components of such optical fiber probe include a small lens and a beam director, where both provide a focused optical beam directing it to a location of interest through a guide-wire. Traditionally, this type of small optical probe has been implemented by attaching a small radial graded refractive index (GRIN) glass rod lens or called SELFOC lens with a size range of $0.25-1.0 \mathrm{~mm}$ and a tiny glass micro-prism to a single mode fiber (SMF) with optical adhesive or optical epoxy (Li et al., 2000). However, the gluing of a separate small lens and a tiny prism to a fiber is a complex fabrication process that results in a low quality optical interface. A new probe design that uses optical fiber lenses, e.g., fiber GRIN lens or fiber ball lens, has been proposed (Swanson et al., 2002; Shishkov et al., 2006). The main advantage of fiber lenses over conventional glass lenses are their small size, ability to auto-align to a fiber, thus creating a fusion-spliced interface with low loss, low back-reflection, and high mechanical integrity. In addition, a beam director can be easily attached to the fiber lenses by the fusion-splice of a polished fiber spacer and direct polish on the ball lens. Beam quality of a fiber-optic probe is crucial for the imaging system. Ideal characteristics of a fiber-optic probe include a high Gaussian beam intensity profile, an appropriate intensity-distance shape, high flexibility, and low optical aberration and loss. 
Swanson et al.and Shishkov et al. proposed the fiber based optic probes design, but presented the variations of probe structure instead of the characteristics of their performance (Swanson et al., 2002; Shishkov et al., 2006). Reed et al. demonstrated the usage of such probes with emphasis on their insertion loss only (Reed et al., 2002). Yang et. al. (Yang et al., 2005b), Jafri et. al. (Jafri et al., 2005), and Li et. al. (Li et al., 2006) reported OCT images without detailed characterization of the used fiber lens based probes. We recently reported design, fabrication, and characterization of the fiber probes with comparison in detail the actual optical performance of a fiber-based optic probe with modeling results (Mao et al., 2007; Mao et al., 2008).

In the second section in this chapter, we present theoretical and experimental results for a $3 \times 3$ Mach-Zehnder quadrature interferometer to acquire a complex interferometric signal for SS-OCT system. We introduce a novel unbalanced differential detection method to improve the overall utilization of optical power and provide simultaneous access to the complementary phase components of the complex interferometric signal. No calculations by trigonometric relationships are needed. We compare the performance for our setup to that of a similar interferometer with a commonly used balanced detection technique. We demonstrate complex conjugate artifact suppression of $27 \mathrm{~dB}$ obtained in a swept-source optical coherence tomography using our unbalanced differential detection. We show that our unbalanced differential detection has increased signal-to-noise ratio by at least $4 \mathrm{~dB}$ comparing to a commonly used balanced detection technique. In the third section, we demonstrate a Fourier-domain mode-lock (FDML) wavelength-swept laser based on a polygon scanner filter and a high-efficiency semiconductor optical amplifier. Peak and average output powers of $98 \mathrm{~mW}$ and $71 \mathrm{~mW}$, respectively, have been achieved without an external amplifier, while the wavelength was swept continuously in a full wavelength of 113 $\mathrm{nm}$ at center wavelength of $1303 \mathrm{~nm}$. A unidirectional wavelength sweeping rate of 7452 $\mathrm{nm} / \mathrm{ms}(65.95 \mathrm{kHz}$ repetition rate) was achieved by using a 72 facet polygon with a rotational rate, $\mathrm{R}$, of 916 revolutions per second. The instantaneous linewidth of this laser is $0.09 \mathrm{~nm}$, which corresponds to a coherence length of $16 \mathrm{~mm}$. We also construct an OCT system that uses our laser source where we have shown that its parameters are optimized for this application. In the fourth section, we discuss design methods and fabrication techniques of fiber-lens-based optic probes. We compare in detail measured performance with expected theoretical performance. Finally, we demonstrate the images of human skins, animal arterial plaque and heart tissues acquired from our catheter-based complex SS-OCT, which proves our SS-OCT system with fiber catheter is most suitable for the applications of biomedical imaging.

\section{Full Range (Complex) Optical Coherence Tomography System}

\subsection{Theoretical Analysis of the Complex System}

An MZI utilizing a 3×3, two 2x2 fiber couplers, and two differential detectors is shown in Fig. 1. A $90 / 102 \times 2$ fiber coupler is used as a power divider of the light source: $90 \%$ power to the sample and $10 \%$ power to reference arms. This is an advantage of the MZI (Rollins \& Izatt, 1999), which allows more light to the sample arm for compensating the lower reflection of a biological sample in an OCT system. The $3 \times 3$ fiber coupler serves not only as a combiner of the two signals from the sample and reference arms, but also provides three phase related 
output interferometric signals. To form two phase related differential detections, which are necessitated to obtain the real and imaginary parts of the interferometric signal, one of the output ports of the $3 \times 3$ coupler is split using one 50/50 2x2 fiber coupler. Two differential detectors were constructed by combining one output of the $2 \times 2$ coupler and one of the remaining outputs of the $3 \times 3$ coupler. We note that the input signals for these differential detectors are not balanced, but no optical power is lost. For comparison, the different unbalanced differential detection methods with different input power ratios, achieved by adjusting two additional fiber attenuators, are also shown in Fig. 1. When the input power ratio is adjusted to achieve balanced detection (i.e. attenuation $\alpha=0.5$ ), the DC component of the interferometric signal could be dynamically removed, but one third of the optical power would be lost.

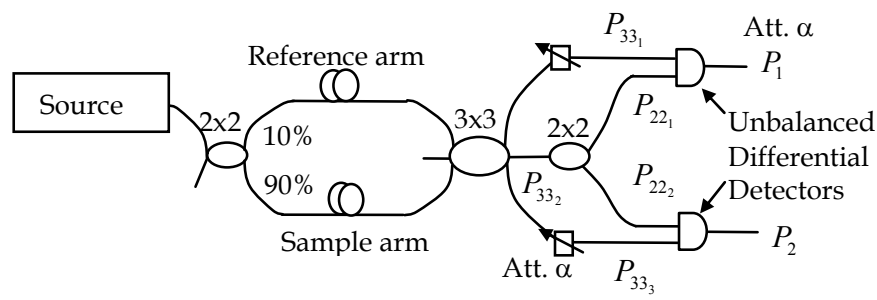

Fig. 1. Mach-Zehnder interferometer using a $3 \times 3$ and two $2 \times 2$ fiber couplers to form two channel unbalanced (attenuation $\alpha=1-0.5$ ) and balanced (attenuation $\alpha=0.5$ ) differential detections for acquiring real and imaginary parts of the interferometric signal.

To analyze our setup we could use transfer matrix descriptions for both $2 \times 2$ and $3 \times 3$ couplers (Sheem 1981; Priest, 1982). The output electric field of a 2x2 coupler, $\mathbf{E}_{\text {out }_{2 \times 2}}=\left[\begin{array}{ll}E_{\text {out }} & E_{\text {out }}\end{array}\right]^{T}$, due to an input electric field, $\mathbf{E}_{\text {in }_{2 \times 2}}=\left[\begin{array}{ll}E_{i n_{1}} & E_{i n_{2}}\end{array}\right]^{T}$, where $T$ denotes matrix transposition, is given by $\mathbf{E}_{\text {out } t_{2 \times 2}}=\mathbf{M}_{2 \times 2} \mathbf{E}_{\mathbf{i n}_{2 \times 2}}$, where

$$
\mathbf{M}_{2 \times 22_{50 / 50}}=\left[\begin{array}{cc}
\sqrt{0.5} & -j \sqrt{0.5} \\
-j \sqrt{0.5} & \sqrt{0.5}
\end{array}\right]
$$

presents the transfer matrix of a 50/50 2x2 coupler, and

$$
\mathbf{M}_{2 \times 22_{9 / 10}}=\left[\begin{array}{cc}
\sqrt{0.9} & -j \sqrt{0.1} \\
-j \sqrt{0.1} & \sqrt{0.9}
\end{array}\right]
$$

is the transfer matrix of a $90 / 102 \times 2$ coupler. Similarly, the output electric field of a $3 \times 3$ coupler could be written as $\mathbf{E}_{\text {out }_{3 \times 3}}=\mathbf{M}_{3 \times 3} \mathbf{E}_{\mathbf{i n}_{3 \times 3}}$, where

$$
\mathbf{M}_{3 \times 3}=\frac{e^{-j 2 K_{\text {cpl }}}}{3}\left[\begin{array}{lll}
1 & 1 & 1 \\
1 & 1 & 1 \\
1 & 1 & 1
\end{array}\right]+\frac{e^{j 2 K_{\text {cpl }}}}{3}\left[\begin{array}{ccc}
2 & -1 & -1 \\
-1 & 2 & -1 \\
-1 & -1 & 2
\end{array}\right]
$$

and $K_{c p l}$ is the coupling coefficient and equals to 0.7 for a $3 \times 3$ coupler with $1 / 3$ power coupling ratio (Sheem 1981$)$.

The operation of a Mach-Zehnder interferometer could be represented by a cascade of the transfer matrices of the couplers and a matrix representing the phase shift between the 
sample and reference arms $\phi$. Let the input electric field and the matrix representing the phase shift between the sample and reference arms be given by $\mathbf{E}_{\text {in }}=\left[\begin{array}{lll}0 & 1 & 0\end{array}\right]^{T}$ and $\mathbf{M}_{\varphi}=\left[\begin{array}{ccc}0 & 0 & 0 \\ 0 & 1 & 0 \\ 0 & 0 & e^{j \phi}\end{array}\right]$, respectively. In our setup, the splitter ratio of the first $2 \times 2$ coupler is $90 / 10$. Therefore, the output electric field, $\mathbf{E}_{33}(\varphi)=\left[\begin{array}{lll}E_{33_{1}}(\phi) & E_{33_{2}}(\phi) & E_{33_{3}}(\phi)\end{array}\right]^{T}$ after the $3 \times 3$ coupler shown in Fig. 1 is calculated by:

$$
\mathbf{E}_{33}(\varphi)=\mathbf{M}_{3 \times 3} \mathbf{M}_{\varphi}(\phi) \mathbf{M}_{2 \times 2} \mathbf{E}_{90 / 10} .
$$

In the case of the $2 \times 2$ coupler after the $3 \times 3$ coupler, if the input electric field is given by $\mathbf{E}_{\mathbf{i n}_{2 \times 2}}(\phi)=\left[E_{33_{2}}(\phi) \quad 0\right]^{T}$, the output electric fields, $\mathbf{E}_{\mathbf{2 2 _ { 1 }}}, \mathbf{E}_{\mathbf{2 2}_{2}}$, are obtained by:

$$
\begin{aligned}
& \mathbf{E}_{22_{1}}(\phi)=\mathbf{M}_{2 \times 2_{50 / 50}} \mathbf{E}_{\mathbf{i n}_{2 \times 2}}(\phi), \\
& \mathbf{E}_{22_{2}}(\phi)=\mathbf{M}_{2 \times 2_{50 / 50}} \mathbf{E}_{\mathbf{i n}_{2 \times 2}}(\phi) .
\end{aligned}
$$

Therefore, the related optical powers $P_{33_{1}}, P_{22_{1}}, P_{22_{2}}$, and $P_{33_{3}}$ specified in Fig. 1 are calculated by:

$$
P \propto \mathbf{E}^{*} \mathbf{E} .
$$

The interferometric signal powers $P_{1}$ and $P_{2}$ from the outputs of the two differential detectors v.s. the attenuation value $\alpha$ and the phase shift between the sample and reference arms $\phi$ are calculated by subtracting the two optical input signal powers of the detectors, respectively, i.e.,

$$
\begin{gathered}
P_{1}(\phi)=\alpha P_{33_{1}}(\phi)-P_{22_{1}}(\phi), \\
P_{2}(\phi)=\alpha P_{33_{3}}(\phi)-P_{22_{2}}(\phi) .
\end{gathered}
$$

The phase differences between the two interferometric signals $P_{1}$ and $P_{2}$ and the related power levels are obtained by graphing their function curves vs. $\phi$.

The real $\left(P_{R E}\right)$ and imaginary $\left(P_{I M}\right)$ part signals, e.g. quadrature components $\left(0^{\circ}\right.$ and $\left.90^{\circ}\right)$, are formed from the interferometric signals $P_{1}$ and $P_{2}$ acquired at two differential detectors using the following trigonometric equations (Choma et al., 2003):

$$
P_{R E}(\phi)=P_{1}(\phi), \quad P_{I M}(\phi)=\frac{P_{1}(\phi) \cos (\Delta \phi)-P_{2}(\phi)}{\sin (\Delta \phi)}
$$

where, $\Delta \phi$ is the phase difference between the interferometric signals $P_{1}$ and $P_{2}$. The wavelength dependent power splitting ratios of the fiber couplers were neglected in this work for simplicity. SS-OCT A-scans with resolved complex conjugate artifact were obtained by inverse Fourier transformation (IFT) of the complex signal $P_{R E}+j P_{I M}$. 


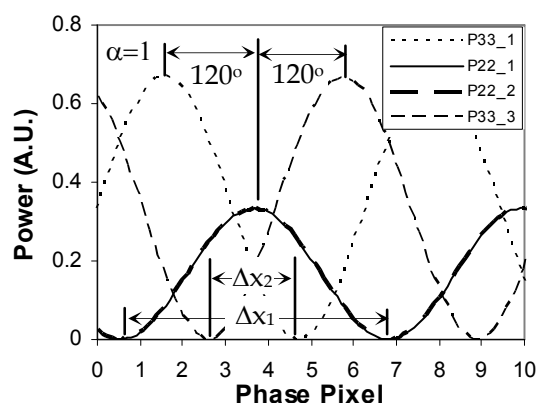

(a)

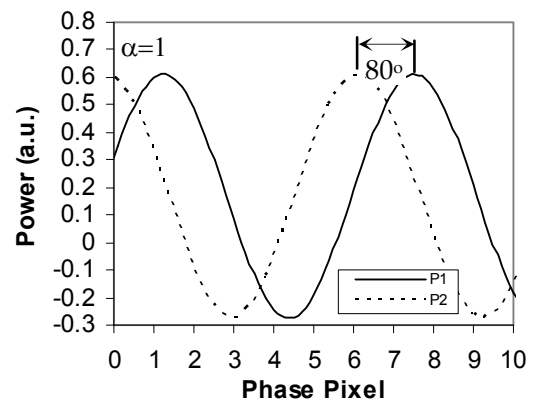

(c)

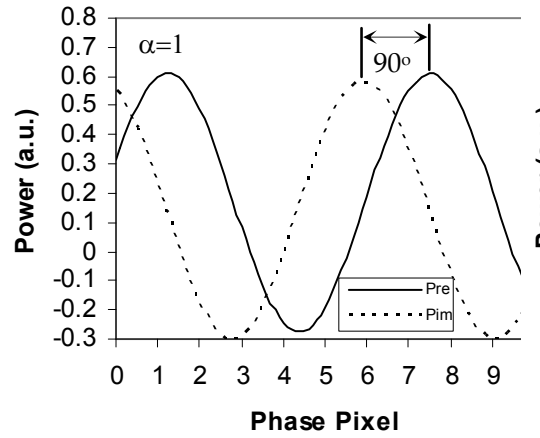

(e)

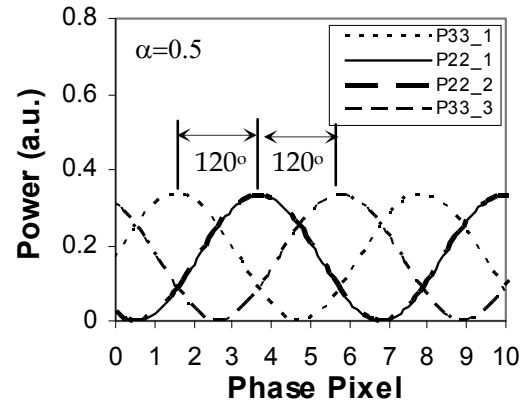

(b)

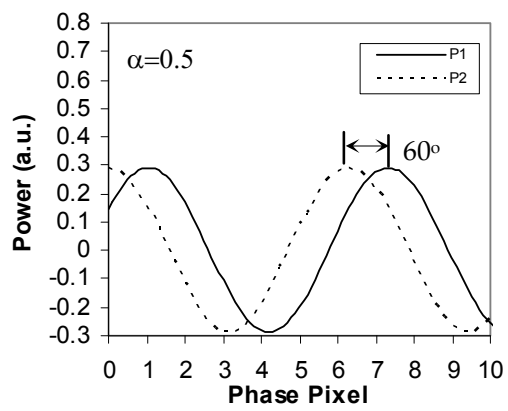

(d)

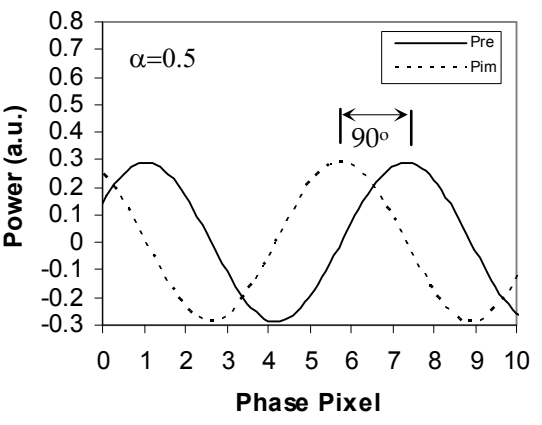

(f)

Fig. 2. Theoretical waveforms before $(a, b)$ and after $(c, d)$ the differential detection and after the quadrature signal calculations $(\mathrm{e}, \mathrm{f})$ when the attenuation $\alpha=1(\mathrm{a}, \mathrm{c}, \mathrm{e})$ and $0.5(\mathrm{~b}, \mathrm{~d}, \mathrm{f})$, where $\alpha=1.0$ and 0.5 correspond to the unbalanced by a factor of 2 and balanced configurations, respectively.

Fig. 2 shows theoretical waveforms when the attenuation $\alpha=1$ (a, c, e) and $0.5(\mathrm{~b}, \mathrm{~d}, \mathrm{f})$, where $\alpha=1.0$ and 0.5 correspond to the unbalanced by a factor of 2 and balanced 
configurations, respectively. The waveforms shown in Fig.2 (a, b) and (c, d) are calculated before and after the differential detections, respectively, while the waveforms shown in Fig.2 (e, f) are calculated using the Eq. 8. The phase difference $\Delta$ was calculated using $\Delta=$ $2 \pi \Delta \mathrm{x}_{2} / \Delta \mathrm{x}_{1}$, where $\Delta \mathrm{x}_{1}$ and $\Delta \mathrm{x}_{2}$ are the phase pixel intervals of a period and a difference of the two signals, respectively, as illustrated in Fig. 2 (a). The phase differences of 120 between the two input detector signals were obtained for both $\alpha=0.5$ and 1.0 configurations as shown in Fig. $2(a, b)$. This is due to the phase difference resulting from the $3 \times 3$ coupler. Because of the power difference of the input signals, the amplitude levels and the phase relationships of the output signals would be different in the two setups as shown in Fig. 2.

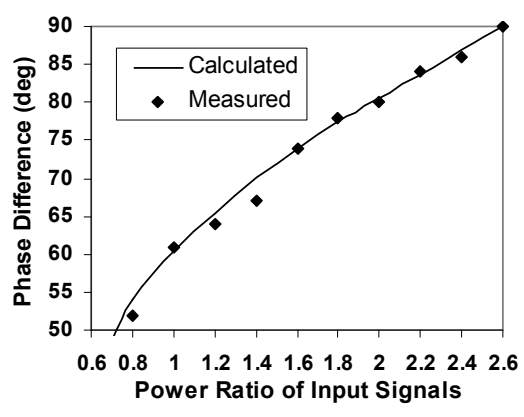

(a)

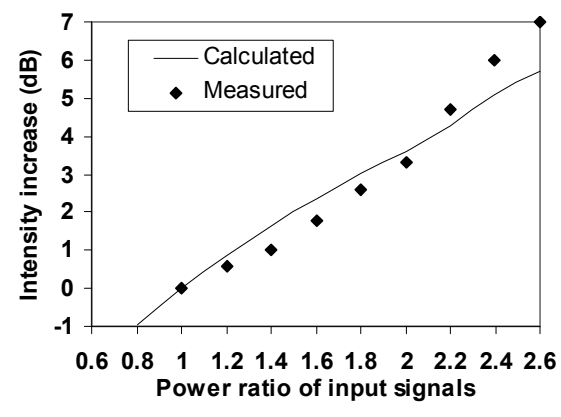

(b)

Fig. 3. Theoretical phase difference (a) and intensity increase (b) of the output signals for the unbalanced configuration from the balanced configuration verses power ratio of the two detector input signals, respectively. The experimental results are shown as the points.

Fig. 3 (a) and (b) show theoretical and experimental results of the phase difference and intensity increase of the output signals, $\mathrm{P}_{1}$ and $\mathrm{P}_{2}$ for the unbalanced configuration from the balanced configuration versus input power ratios of the detectors, respectively. The phase differences of the output signals are $60^{\circ}$ and $80^{\circ}$ when power ratios of input signals are 1 (balanced configuration) and 2 (unbalanced configuration), respectively, obtained both in theory and experiment. The intensities of the output signals increase $3 \mathrm{~dB}$ when the power ratio of input signals is 2 (unbalanced configuration) comparing to the power ratio of input signals is 1 (balanced situation). The input power ratios from 2 to 2.6 for the detectors could be obtained when the coupling ratio of $3 \times 3$ was not exactly $1 / 3$. From the results shown in Fig. 3 (a), a $90^{\circ}$ phase difference between the detector outputs $P_{1}$ and $P_{2}$ could be obtained if the input power ratio was 2.6, therefore, no calculations by the trigonometric relationships are needed for obtaining the quadrature signals.

\subsection{Method of the Complex OCT System}

Fig. 4 shows the experimental setup of the instantaneous complex conjugate resolved sweptsource OCT system using the $3 \times 3$ Mach-Zehnder interferometer topology with the unbalanced (no attenuators) and balanced (with attenuators) differential detection schemes. The system consisted of a $2 \times 2$ single-mode fiber coupler with $90 / 10$ coupling ratio as the 
input coupler and a $3 \times 3$ fiber coupler with coupling ratios of $0.39 / 0.29 / 0.32$, a $50 / 502 \times 2$ fiber couplers and two variable fiber attenuators as the output couplers forming the dualchannel balanced detection.

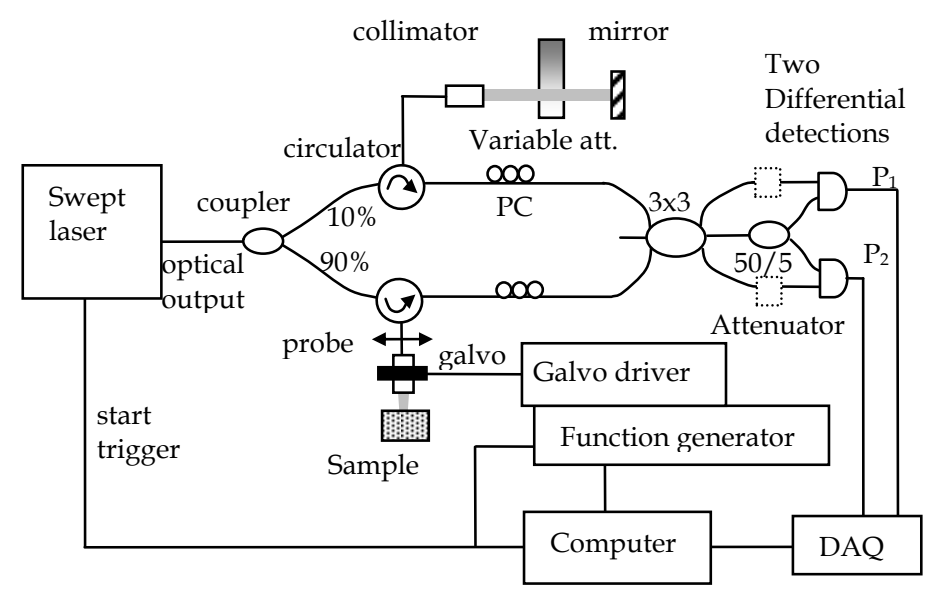

Fig. 4. Schematic diagram of the instantaneous complex conjugate resolved swept-source OCT system using the $3 \times 3$ Mach-Zehnder interferometer (MZI) topology with unbalanced (no attenuators) and balanced (with attenuators in the doted line) differential schemes. The coupler ratios of the $3 \times 3$ coupler are $0.39 / 0.29 / 0.32$.

The swept source (HSL2000-HL, Santac) used in the system had a central wavelength of $1320 \mathrm{~nm}$ and a full scan wavelength range of $110 \mathrm{~nm}$, which was sweeping linearly with optical frequency with a linearity of $0.2 \%$. The average output power and coherence length of the swept source was $12 \mathrm{~mW}$ and $10 \mathrm{~mm}$, respectively. A repetition scan rate of $20 \mathrm{kHz}$ was used in our system and the related duty cycle was $68 \%$. The output light from the swept laser source was launched into the first coupler and then divided into the sample arm with $90 \%$ power and reference arm with $10 \%$ power by two fiber circulators. The reference arm was arranged with a fiber collimator and a mirror. A variable attenuator was inserted between the collimator and mirror for adjusting the optical power on reference arm to achieve the higher sensitivity. The light was illuminated to a sample through the lensed single mode fiber probe with working distance (focus distance to lens surface in air) of 1.1 $\mathrm{mm}$, depth of field (twice the Rayleigh range in air) of $1.1 \mathrm{~mm}$, and 1/ $\mathrm{e}^{2}$ spot diameter (transverse resolution) of $27 \mu \mathrm{m}$ which will be described in the third section in this chapter. A galvanometer (Blue Hill Optical technologies) scanner scanned the fiber probe light transversely on the sample up to $4 \mathrm{~mm}$ at $20 \mathrm{~Hz}$ with 1000 transverse pixels. The total optical power illuminating on the sample was approximately $10 \mathrm{~mW}$. Two polarization controllers (PC) in both reference and sample arms were used for adjustment to match the polarization state of the two arms. The two-pair output signals from the output couplers were detected with two-pair photodiodes to obtain quadrature signals. Two differential photo-detectors (PDB150C, Thorlabs) were used with adjustable bandwidth. A 3 dB 
bandwidth of $50 \mathrm{MHz}$ was used in our system. The two detector outputs were digitized using a data acquisition card (DAQ) (PCI 5122, National Instruments) with 14-bit resolution and acquired at a sampling speed of $100 \mathrm{MS} / \mathrm{s}$. The swept source generated a start trigger signal that was used to initiate the function generator for the galvo scanner and initiate the data acquisition process for each A-scan. Because the swept source was linearly swept with wave-number $\mathrm{k}$, A-scans data with resolved complex conjugate artifact were obtained by a direct inverse Fourier transformation (IFT) from direct DAQ sampling data without any resampling process.

\subsection{Results and Discussion of Complex OCT system}

The performance of the complex conjugate ambiguity resolution in our 3x3 Mach-Zehnder SS-OCT system with the unbalanced configuration could be quantified by comparing the complex conjugate resolved A-scans with the unresolved A-scans. Measurements were taken using a $-55 \mathrm{~dB}$ reflector including coupling loss in the sample arm as shown in Fig. 4 . The reference mirror was adjusted to a position such that the difference in optical path length between the two interferometer arms was $500 \mu \mathrm{m}$. The extra background noise was subtracted by measuring the reference arm signals with the sample arm blocked.

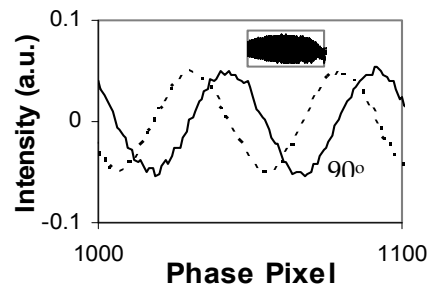

(a)

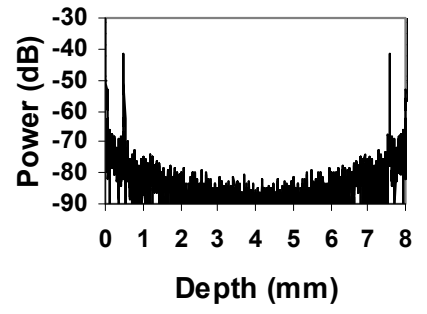

(b)

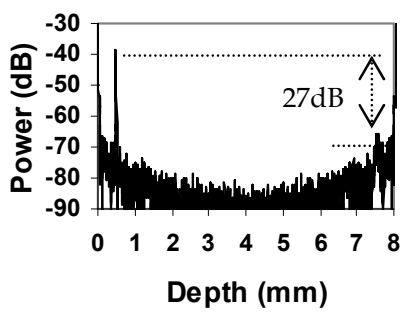

(c)

Fig. 5. The experimental results of the complex conjugate artifact resolution with our $3 \times 3$ MZI SS-OCT in the unbalanced differential detection with $3 \times 3$ coupler ratio of $0.39 / 0.29 / 0.32$ in $500 \mu \mathrm{m}$ path length difference of the sample and reference arms. (a): Measured interferometric signals of the output $\mathrm{P}_{1}$ and $\mathrm{P}_{2}$ on two detectors with phase shift of $90^{\circ}$. Inset: Measured full interferometric signals of the output $P_{1}$ and $P_{2}$. (b): A-scan signals obtained by IFT from a single detector include the complex conjugate artifacts. (c): A-scan signals obtained by IFT directly from the output signals at the two detectors with suppressions of the complex conjugate peaks of $27 \mathrm{~dB}$.

Fig. 5 (a) shows measured waveforms of the output signal $P_{1}$ and $P_{2}$ from our unbalanced SS-OCT system with $3 \times 3$ port configuration as coupler ratio of $0.39 / 0.29 / 0.32$, i.e., we connected the lowest power port of the $3 \times 3$ coupler to the $2 \times 2$ coupler. The input power ratios in this unbalanced setup were 2.8 and 2.4 for the two detectors, respectively. We noticed from the full interferometric signals of the output $P_{1}$ and $P_{2}$ shown in the inset of the Fig. 5 (a) that the DC values of the waveforms in the unbalanced system were removed by the high-pass filter of the detectors. Because a phase difference of $90^{\circ}$ between the interferometric signals $P_{1}$ and $P_{2}$ was obtained, the data of measured $P_{1}$ and $P_{2}$ was automatically become quadrature signals. Therefore, a minimized complex conjugate 
artifact was obtained after performing a complex Fourier transform operation directly from the two outputs. Fig. 5 (b) shows the A-scan at $500 \mu \mathrm{m}$ depth obtained by inverse Fourier transform of a single detector include the complex conjugate artifact. The symmetric reflective peaks were in both positive and negative sides. Fig. 5 (c) shows the complex conjugate resolved A-scan obtained by taking the inverse Fourier transform directly from the output quadrature signals $P_{1}$ and $P_{2}$. SNR and suppression of the complex conjugate peaks of $52 \mathrm{~dB}$ and $27 \mathrm{~dB}$ was obtained at about $500 \mu \mathrm{m}$ depths in our unbalanced differential detection SS-OCT system when the $55 \mathrm{~dB}$ reflector in the sample arm was used. Therefore, the measured sensitivity of $107 \mathrm{~dB}$ was obtained in our system. We found the sensitivity obtained from the system with the unbalanced configuration was increased by 4 $\mathrm{dB}$ compared to the system with the balanced configuration. This is due to better utilization of optical power with our unbalanced differential detection technique.

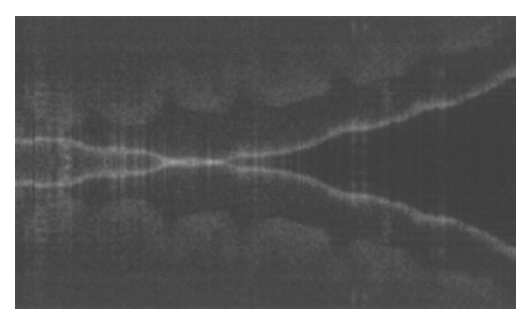

(a)

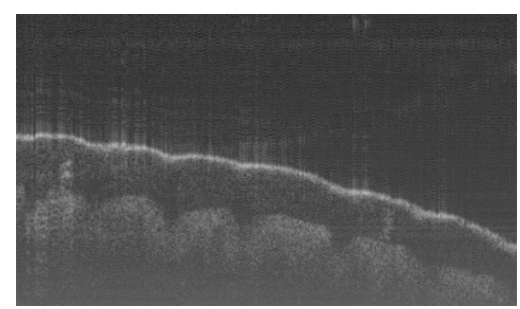

(b)

Fig. 6. In vivo images of human finger tip acquired by our full range swept-source optical coherence tomography using the $3 \times 3$ Mach-Zehnder interferometer with unbalanced differential detection technique. (a): the image was generated using only a single detector. (b): the complex signals were used.

Fig. 6 shows in vivo images of a human finger tip acquired by our full range swept-source optical coherence tomography using the $3 \times 3$ Mach-Zehnder interferometer with unbalanced differential detection technique. The resolutions of the axial and lateral are $10 \mu \mathrm{m}$ and $27 \mu \mathrm{m}$, respectively. The pixel size of the images is $800 \times 900$ in correspond to the image size of $3 \times 4 \mathrm{~mm}$. In Fig. 6 (a), the image was generated using only a single detector, and demonstrates the folded artifact images. In Fig. 6 (b), the complex signal was used demonstrating artifact-free imaging over a depth range of $3 \mathrm{~mm}$.

\section{High Performance Wavelength-Swept Laser for Optical Coherence Tomography}

\subsection{Optical Filter Design for Swept Laser}

Fig. 7 shows a schematic diagram of the FDML wavelength swept laser with a long fiber ring cavity. A SOA is used as the gain medium in the ring cavity which has a central wavelength of $1300 \mathrm{~nm}$. The SOA is considered the most suitable gain medium for OCT applications: high small-signal gain, broadband gain spectra, and high relaxation resonance frequencies. The wavelength selection was achieved by a polygon-based high-speed narrowband optical scanning filter. The unidirectional wavelength sweep of a polygon 
scanner, as opposed to the inherently bidirectional Fabry-Perot filter, is better matched to the gain properties of an SOA. A mode-locked laser resonator was implemented using a long single mode fiber (SMF). The polygon-based reflection-type scanning narrow-bandpass filter (Yun et al., 2003) includes an optical fiber collimator, an optical diffraction grating, an afocal telescope, a polygon scanner, and a planar mirror. A collimated Gaussian beam with a broad optical spectrum from the SOA was first spread by the optical grating and then converged to the polygon by the telescope. The telescope, made of two achromatic doublets, controls both the beam size and angles. A planar mirror was placed after the polygon to reflect only the spectral component with normal incidence to the fiber. We note that the sweeping angle of the intermediate reflection from the polygon facet doubles the polygon's effective rotation angle, so that the free spectral range (FSR) of this filter is twice than when the polygon simply retro-reflects the light back to the telescope. The orientation of the grating incidence angle and the rotation direction of the polygon facet determine the direction of the wavelength tuning. The arrangement in Fig. 7 produced a unidirectional increase of the wavelength that resulted in higher optical power output. An optical fiber circulator was used to couple the light between the ring cavity and the filter. Three fiber polarization controllers were placed before the SOA, the circulator and the grating to optimize their polarization, respectively (Mao et al., 2009). The CW wavelength-swept light was coupled out of the cavity by a fiber coupler positioned after the SOA.

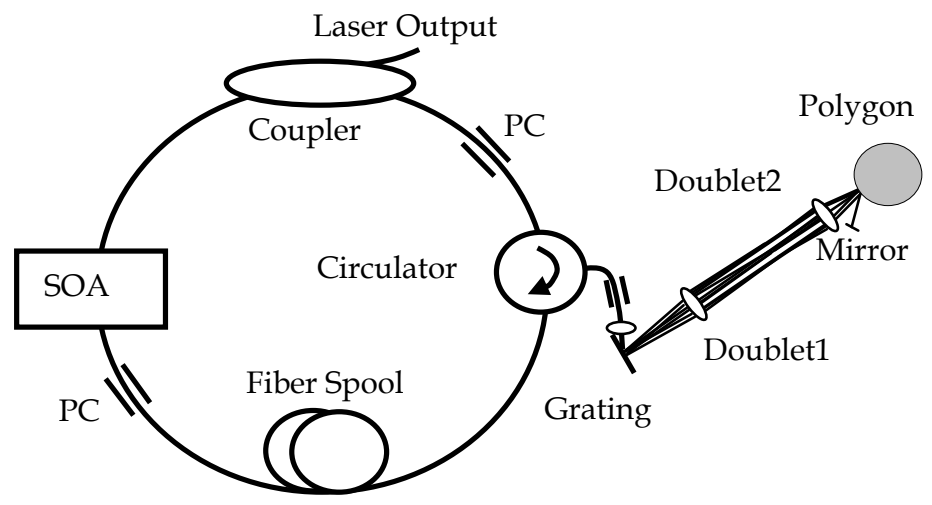

Fig. 7. Schematic diagram of a FDML wavelength-swept laser shown with a ZEMAX raytracing of the polygon-based narrow-band filter.

The parameters of the polygon scanning filter and its components were investigated by using a commercial optical modeling software ZEMAX (ZEMAX Development Corp., WA, USA). The ray-tracing layout of the optical filter is shown in Fig. 7 where a non-sequential ZEMAX component was used to model the polygon scanner. As the polygon was rotated clockwise by one facet, one full cycle of the continuous spectrum was swept. To achieve an optical filter with a FWHM linewidth of $0.16 \mathrm{~nm}$, central wavelength of $1305 \mathrm{~nm}$, free spectral range (FSR) of $110 \mathrm{~nm}, 100 \%$ duty cycle, minimum beam clipping and maximum coupling efficiency, the following optical components were chosen: a fiber collimator with $10 \mathrm{~mm}$ focal length and $1 / \mathrm{e}^{2}$ beam width of $1.89 \mathrm{~mm}$, a grating with a groove frequency of $830 / \mathrm{mm}$ and an incident angle of $69^{\circ}$, two achromatic doublets with focal lengths of $75 \mathrm{~mm}$ 
and $40 \mathrm{~mm}$, a polygon scanner with 72 facets and a facet area of $6.35 \times 2.77 \mathrm{~mm}^{2}$ (Mao et al., 2009). A summary of the input parameters and simulation results are listed in Table 1 . The observed $0.5 \mathrm{~mm}$ chromatic focal shift after F1 on both end of the spectrum wavelength ends were compensated for our double-pass arrangement. A change of the focal spot at the image plane from circular to elliptical at the edge wavelengths, shown in Fig. 8, resulted in a nearly Gaussian output spectrum due to lower coupling efficiencies at those edges.

\begin{tabular}{|c|c|c|c|}
\hline Input Parameter & Value & Simulation Result & Value \\
\hline $\begin{array}{l}\text { Center } \\
\text { wavelength }\end{array}$ & $1305 \mathrm{~nm}$ & Collimate beam width $\left(1 / \mathrm{e}^{2}\right)$ & $1.89 \mathrm{~mm}$ \\
\hline $\begin{array}{l}\text { Input fiber, } \\
\text { core \& NA }\end{array}$ & $\begin{array}{l}\text { Single mode } \\
9 \mu \mathrm{m} \& 0.11 \\
\end{array}$ & Spectral sweeping range & $\pm 55 \mathrm{~nm}$ \\
\hline Collimate lens & $\begin{array}{c}f=10 \mathrm{~mm} \\
\phi=6 \mathrm{~mm}\end{array}$ & FWHM bandwidth at central & $0.16 \mathrm{~nm}$ \\
\hline $\begin{array}{l}\text { Grating density } \\
\text { incidence angle }\end{array}$ & $\begin{array}{c}830 \mathrm{~g} / \mathrm{mm} \\
\phi=69 \mathrm{deg}\end{array}$ & $\begin{array}{l}\text { Diverging angle after grating } \\
\text { at } 1250 \text { and } 1360 \mathrm{~nm} \text { to central }\end{array}$ & $\begin{array}{l}-2.637^{\circ} \\
+2.656^{\circ}\end{array}$ \\
\hline Doublet 1 & $\begin{array}{c}F 1=75 \mathrm{~mm} \\
\phi=25.4 \mathrm{~mm}\end{array}$ & $\begin{array}{l}\text { Converging angle at polygon } \\
\text { at } 1250 \text { and } 1360 \mathrm{~nm} \text { to central }\end{array}$ & $\begin{array}{l}+4.944^{\circ} \\
-4.979 \circ\end{array}$ \\
\hline Doublet 2 & $\begin{array}{l}F 2=40 \mathrm{~mm} \\
\phi=25.4 \mathrm{~mm}\end{array}$ & 1/e $\mathrm{e}^{2}$ Beam width at Polygon & $2.78 \mathrm{~mm}$ \\
\hline $\begin{array}{l}\text { Polygon facet } \\
\text { number }\end{array}$ & 72 & $\begin{array}{l}\text { Chromatic focal shift after F1 } \\
\text { at } 1250 \text { and } 1360 \mathrm{~nm} \text { to central }\end{array}$ & $\begin{array}{l}0.50 \mathrm{~mm} \\
0.51 \mathrm{~mm}\end{array}$ \\
\hline $\begin{array}{l}\text { Polygon angle } \\
\text { per facet }\end{array}$ & $5^{\circ}$ & $\begin{array}{l}\text { Image chromatic focal shift at } \\
1250 \text { and 1360nm to central }\end{array}$ & $\begin{array}{l}<0.1 \mathrm{~mm} \\
<0.1 \mathrm{~mm}\end{array}$ \\
\hline Polygon radius & $31.75 \mathrm{~mm}$ & $\begin{array}{l}\text { Image spot size at 1250, } \\
1305 \text {, and } 1360 \mathrm{~nm}\end{array}$ & $\begin{array}{c}120 \times 10 \mu \mathrm{m}^{2} \\
2.6 \times 2.6 \mu \mathrm{m}^{2} \\
110 \times 9 \mu \mathrm{m}^{2}\end{array}$ \\
\hline $\begin{array}{l}\text { Polygon facet } \\
\text { area }\end{array}$ & $6.35 \times 2.77 \mathrm{~mm}^{2}$ & $\begin{array}{l}\text { Coupling efficiency at } 1250, \\
1305, \text { and } 1360 \mathrm{~nm}\end{array}$ & $\begin{array}{c}7 \% \\
38 \% \\
8 \% \\
\end{array}$ \\
\hline
\end{tabular}

Table 1. The parameters of the polygon scanning filter obtained from ZEMAX simulation.

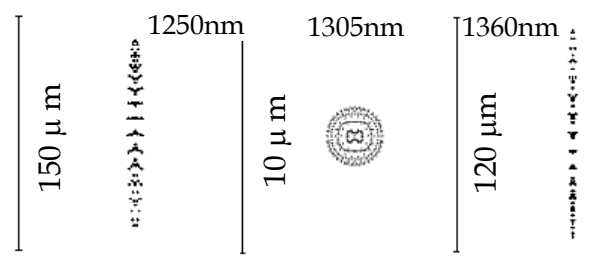

Fig. 8. Imaging spot size at $1250 \mathrm{~nm}$ (left), $1305 \mathrm{~nm}$ (central), and $1360 \mathrm{~nm}$ (right) of the filter from ZEMAX simulation.

The diffraction grating equation is given by (Hecht, 1979), $\lambda=(\sin \alpha+\sin \beta) / T$, where $\lambda$ is the wavelength, $T$ is the groove frequency, and $\alpha$ and $\beta$ are the incident and the diffracted 
angles of the beam, respectively, with respect to the normal axis of the grating. The sweeping wavelength $(d \lambda)$ could be expressed as (Yun et al., 2003):

$$
d \lambda=(1 / T) \cos \beta_{0}\left(F_{2} / F_{1}\right) d \theta
$$

where $d \theta$ is the sweeping angle of the polygon scanner and $\beta_{0}$ is the angle between the optical axis of the telescope and the grating's normal. Since $d \theta=2 \pi R d t$ for a polygon scanner, we could get a linear relationship between the sweeping wavelength and the sweeping time by integrating Eq. (9):

$$
\lambda(t)=\lambda_{1}+\frac{4 \pi \cos \beta_{0} F_{2} R}{T F_{1}} t
$$

Our design results in a wavelength sweeping rate of $7.31 \mathrm{~mm} / \mathrm{s}$.

\subsection{Swept Laser Construction}

A Fourier-domain mode-lock wavelength-swept laser based on polygon scanning filter and semiconductor optical amplifier was constructed. A high efficiency InP/InGaAsP quantum well SOA (BOA 1132, Covega) was used as the laser gain medium with a saturation output power of $19 \mathrm{dBm}$, small signal gain of $30 \mathrm{~dB}$ and FWHM bandwidth of $93 \mathrm{~nm}$. The material structure of the quantum well active region of the SOA was optimized to increase the output power and bandwidth. A 72-facet polygon scanner (SA34, Lincoln Laser) that optimized for ultra-high speed stability was used. Two achromatic doublets (AC254-C, Thorlabs) were used to construct the telescope system. A $3.1 \mathrm{~km}$ optical fiber was inserted into a $5.1 \mathrm{~m}$ ring laser cavity as a delay line fiber to synchronize a repetition rate of $65.95 \mathrm{kHz}$ of the polygon filter, which corresponds to a round-trip propagation time in the cavity of $15.16 \mu \mathrm{s}$. Using this setup, we could alternate between mode-locked and short cavity laser operation. In the mode-locked configuration, we used standard single mode fiber with zero dispersion around $1300 \mathrm{~nm}$ (SMF-130V, Prime Optical Fiber Corporation). The maximum mismatches in the roundtrip times of the different wavelengths due to dispersion and fiber birefringence were $1.1 \mathrm{~ns}$ and $0.4 \mathrm{ps}$, respectively. These values were calculated using a dispersion coefficient of $3.1 \mathrm{ps} \mathrm{km}^{-1} \mathrm{~nm}^{-1}$ and polarization mode dispersion coefficient of $0.2 \mathrm{ps} \mathrm{km}-1 / 2$ of the used SMF-130V fiber. Therefore, these small time mismatches could be neglected. Three fiber polarization controllers for individually aligning polarization states of SOA, fiber circulator and grating were found to be necessary for obtaining maximum optical power. A fiber spool with 6 inch mandrel size was used in the cavity for the $3.1 \mathrm{~km}$ long SMF. We noticed that a fiber spool with 3 inch mandrel could reduce the optical power and flutter the laser spectrum, resulting in a reduction of the $3 \mathrm{~dB}$ bandwidth. This spectral fluctuation could be due to the inconsistent birefringence caused by the small bending radius of the long fiber. The ratio of the output coupler of $60 / 40$ was used $(60 \%$ of the power is coupled out), which was optimized to get the maximum optical output power.

\subsection{Results and Discussions of the Swept Laser}

The time-averaged normalized spectrum emitted from our FDML swept laser, measured by an optical spectrum analyzer (OSA) in peak-hold mode with a resolution of $1 \mathrm{~nm}$, is shown in Fig. 9 (a). On the same graph we show a fitting of the measured spectrum to a Gaussian function, dashed line, and the spectrum obtained by ZEMAX simulations, dotted line. This 
simulated spectrum is the product of the filter spectrum (due to different filter coupling efficiencies at different wavelengths) and the spontaneous emission spectrum of the used SOA. A full sweeping wavelength range of $113 \mathrm{~nm}$ and FWHM bandwidth of $90 \mathrm{~nm}$ at central wavelength of $1303 \mathrm{~nm}$ measured from our swept laser were obtained. We note that the shape of the measured spectrum is close to a Gaussian distribution. This bandwidth of the swept laser would correspond to $8.3 \mu \mathrm{m}$ axial resolution in the air in OCT. The measured sweeping FWHM bandwidth of the FDML swept laser was 1.43 times higher than the simulated spectrum. This increase in bandwidth is due to higher optical gains in the smaller signal range at the edge wavelengths than the central wavelength. While keeping the polygon static, two spectra were measured using the highest resolution of the OSA (0.01 $\mathrm{nm})$ at the center wavelength of $1303 \mathrm{~nm}$ and at the dual-edge wavelengths of $1248.0 \mathrm{~nm}$ and $1358.2 \mathrm{~nm}$, shown in Fig. 9 (b) in addition to the dynamic spectrum for comparison. A distance of $110.2 \mathrm{~nm}$ between the two simultaneous existing peaks corresponds to the FSR of the filter, which is in good agreement with the design specifications of FSR of $110 \mathrm{~nm}$. We also note that the static spectra of our FDML swept laser had a FWHM linewidth of 0.015 $\mathrm{nm}$ at the central wavelength as shown in the inset of the Fig. 9 (b). The measured filter linewidth was $0.17 \mathrm{~nm}$ at the same wavelength as shown in the Fig. 9 (c). We found the measured laser linewidth was 11.3 times smaller than that of the filter. The linewidth of filter in the edge wavelength range increased which could be caused by the off-axis effect of the doublet; however, this effect was diminished by the FDML method as shown in Fig. (c). Fig. 9 (d) shows average output power of the swept laser versus the injection current of the SOA, which shows the laser threshold current is $100 \mathrm{~mA}$.

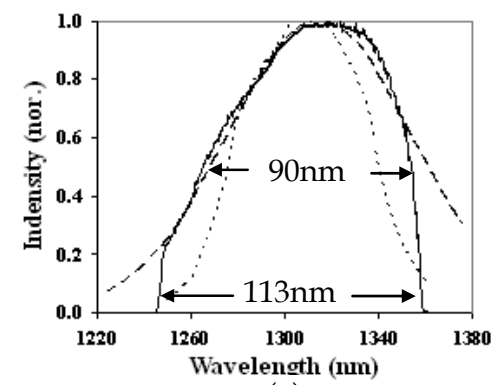

(a)

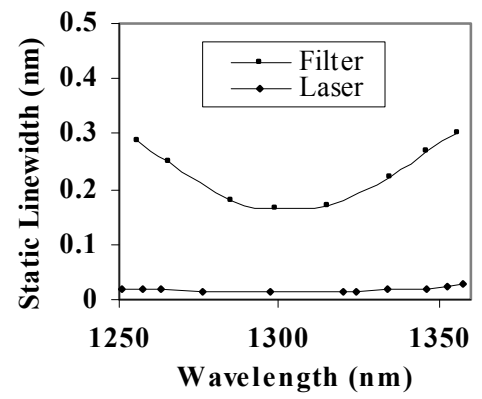

(c)

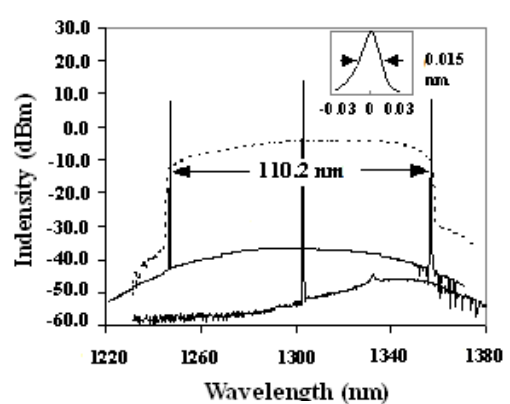

(b)

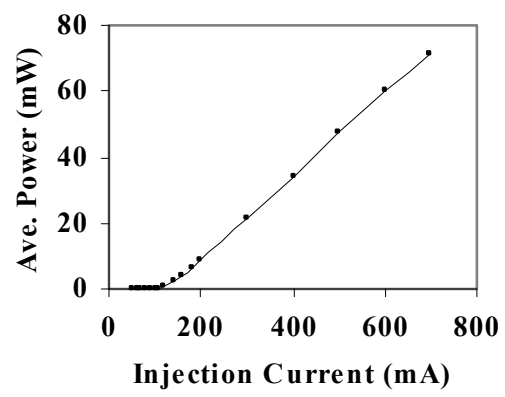

(d)

Fig. 9. (a) Measured normalized spectra of our FDML wavelength-swept laser in solid line, a 
Gaussian fitting in dashed line, and simulated spectrum in dotted line. (b) Measured spectra in $\mathrm{dBm}$ scale with two static spectra while keeping polygon stay at the center wavelength of $1303 \mathrm{~nm}$ and the dual-edge wavelengths of $1248.0 \mathrm{~nm}$ and $1358.2 \mathrm{~nm}$ in solid lines. Inlet: the linear static spectrum at $1303 \mathrm{~nm}$. (c) Measured static linewidth of the FDML swept-laser in comparison with measured linewidth of the polygon filter. (d) Output power versus injection current of the SOA.

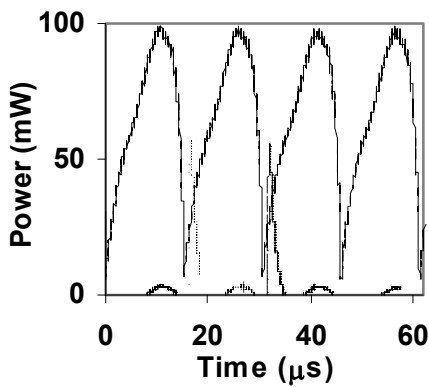

(a)

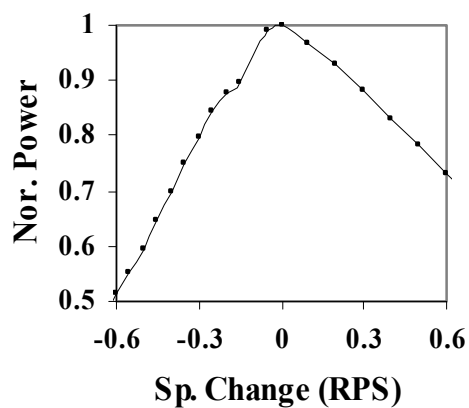

(c)

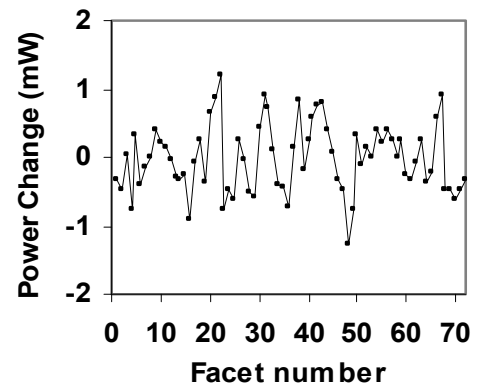

(b)

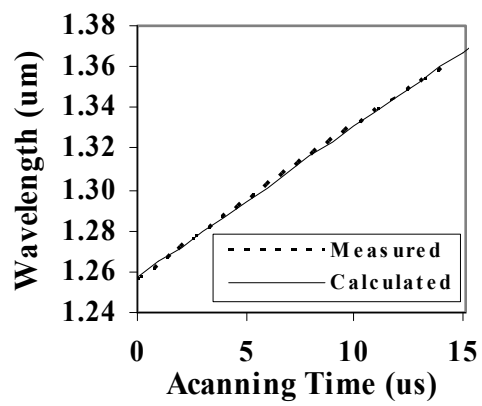

(d)

Fig. 10. (a) Oscilloscope trace of the swept laser output with four tuning cycles at a repetition rate of $65.95 \mathrm{kHz}$ in solid line and a start trigger signal in dashed line. (b) Optical power change versus facet number of the polygon. (c) The normalized output power as a function of polygon speed change. (d) Theoretical and experimental wavelength swept versus time.

Fig. 10 (a) shows the measured output power of our FDML swept laser over four wavelength scans using an oscilloscope. The peak power and the average power emitted by the laser were $98 \mathrm{~mW}$ and $71 \mathrm{~mW}$, respectively, corresponding to an SOA injection current of $700 \mathrm{~mA}$. The observed scan duration of $15.16 \mu$ s with a duty cycle of $100 \%$ correspond to a repetition rate of $65.95 \mathrm{kHz}$. A wavelength start trigger signal obtained by a fiber Bragg grating (FBG) is shown in Fig. 10 (a) in dashed line, which is used for wavelength calibration and system start trigger. It will be discussed below. Laser output power change with 72 successive cycle scans were measured as shown in Fig. 10 (b). The maximum optical power change is less than $3 \mathrm{~mW}$, which corresponds to a maximum $3.3 \%$ optical power fluctuation from facet to facet. Fig. 10 (c) shows the measured normalized output power of the laser as a 
function of the polygon rotation speed change. For this measurement, a function generator was used as an external driver to the polygon scanner. The change in the rotation speed of the polygon was obtained by adjusting the output voltage of the function generator. The laser output power was reduced when the scanner speed was either increased or decreased from the specific speed of 916 revolutions per second (RPS). More power reduction was observed when the polygon speed decreased than when it increased. This effect could be caused by the nonlinearities of semiconductor optical amplifier (Bilenca, 2006). Because the speed stability of our polygon is less than $0.02 \%$, i.e., the speed fluctuation is less than \pm 0.1 RPS, the fluctuation in the measured output power caused by the variation of the polygon speed was less than $1.0 \%$.

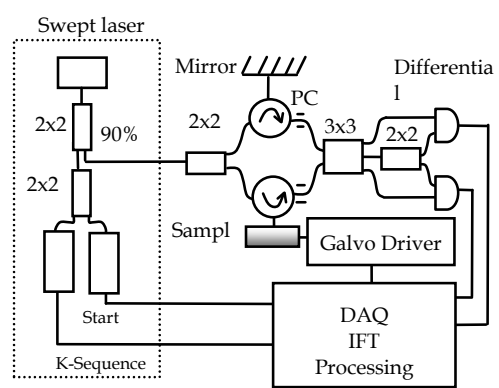

(a)

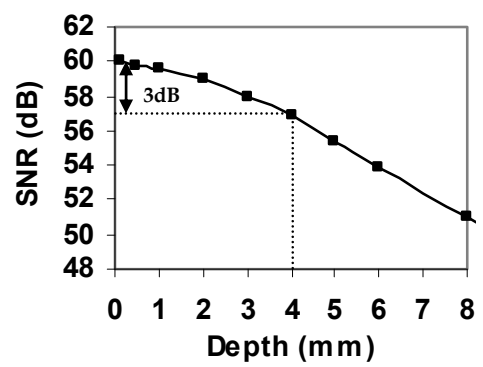

(b)

Fig. 11. (a) Schematic diagram of a complex OCT system using our swept laser with the start trigger and k-sequence. (b) The SNR of the OCT system with out swept source in the different depths.

To further characterize the properties of our swept laser for OCT application, $10 \%$ of the output power of the laser was connected to a $2 \times 2$ coupler to obtain a trigger corresponding to the start wavelength, $\lambda_{1}$, and a sequence of equal differences in the propagation constant $k, \Delta k=2 \pi \Delta \lambda / \lambda^{2}$, where $\Delta \lambda$ is the difference in wavelength. This sequence of equal valual of $k$ is called a $k$-sequence. The start wavelength trigger for the wavelength calibration was implemented using a fiber Bragg grating with central wavelength of $1256 \mathrm{~nm}$ and bandwidth of $0.3 \mathrm{~nm}$, a fiber circulator, and a photo-detector. The sharp rising edge of the start trigger as shown in Fig. 10 (a) in dashed line was used for the trigger of the data acquisition and wavelength calibration in the OCT system. The k-sequence was generated using a Mach-Zhender interferometer (MZI) with an adjustable optical path length difference and a balanced detector. By adjusting the optical path difference to about $8 \mathrm{~mm}$, 1024 peaks at equal values of $k$ were obtained. The measured wavelengths of these 1024 peaks, where $\Delta k$ is equal, versus times was shown in Fig. 10 (d). We found that the measured results were in good agreement with the theoretical results calculated from Eq. 10. Using the remaining $90 \%$ of the optical output power of our swept source, we constructed a complex OCT system using a 3x3 MZI as described in the section 2 and shown in Fig. 11 (a). Measurements at different object depths were taken using a mirror and an attenuator in the sample arm. The extra background noise was subtracted by measuring the reference arm signals with the sample arm blocked. The complex interferometric signals were digitized by 
a data acquisition card (ATS-460, AlazarTech) with 14-bit resolution and acquired at a sampling speed of $125 \mathrm{MS} / \mathrm{s}$. The signal data with equal-k interval were obtained by an interpolation. The start trigger signal was used to initiate the $k$-sequence and the data acquisition process for each A-scan. A-scans with resolved complex conjugate artifact were obtained by a complex inverse Fourier transformation (IFT). The signal noise ratio (SNR) versus depth was obtained by measuring point spread functions (PSF) in the different depth as shown in Fig.11 (b). PSF has a FWHM value of $8.8 \mu \mathrm{m}$, which agrees well with the $8.3 \mu \mathrm{m}$ equivalent to the $3 \mathrm{~dB}$ bandwidth of the spectrum of the laser source. Maximum sensitivity of $114 \mathrm{~dB}$ was obtained by adding the attenuation in the sample arm to the SNR. To estimate the dynamic coherence length of this swept source, we observed a $3 \mathrm{~dB}$ drop in the amplitude of the PSF when the arm length difference was about $4 \mathrm{~mm}$ as shown in Fig. 11 (b). This result corresponds to an optical coherence length of $16 \mathrm{~mm}$ or a linewidth of 0.09 $\mathrm{nm}$. We also observed a drop of $9 \mathrm{~dB}$ in the amplitude of the PSF at an object depth of $8 \mathrm{~mm}$.

\begin{tabular}{|c|c|c|c|c|}
\hline Cavity Length & $\begin{array}{c}3100 \mathrm{~m} \\
\text { (FDML) }\end{array}$ & \multicolumn{3}{|c|}{$5.1 \mathrm{~m}$ short cavity } \\
\hline Repetition Rate $(\mathrm{kHz})$ & 65.95 & 21.5 & 43.8 & 62.1 \\
\hline FWHM Bandwidth $(\mathrm{nm})$ & 90 & 84.6 & 79.0 & 71.4 \\
\hline Coherence Length $(\mathrm{mm})$ & 16 & 14 & 12 & 10 \\
\hline Average Power $(\mathrm{mW})$ & 71 & 62 & 50 & 42 \\
\hline
\end{tabular}

Table 2. Comparison of the performance of our FDML laser with $5.1 \mathrm{~m}$ short cavity laser

To get a better understanding of the operation of our FDML swept laser, we switched the laser to the shorter cavity. Table 2 shows a comparison of the FWHM spectral bandwidth, instantaneous coherence length and average output power of our FDML swept laser operating at $65.95 \mathrm{kHz}$ repetition rate to that of the short cavity swept laser operating at 21.5 $\mathrm{kHz}, 43.8 \mathrm{kHz}$, and $62.1 \mathrm{kHz}$ repetition rates, respectively. In this short cavity laser, when the repetition rate increases, the laser performance is deteriorated, for example, when the repetition rate increases from $21.5 \mathrm{kHz}$ to $62.1 \mathrm{kHz}$, the FWHM spectral bandwidth, instantaneous coherence length and average output power decreases from 84.6 to $71.4 \mathrm{~nm}$, 14 to $10 \mathrm{~mm}$, and $62 \mathrm{~mW}$ to $42 \mathrm{~mW}$, respectively. In comparison, the FWHM bandwidth, coherence length, and average output power of our FDML swept laser reaches $90 \mathrm{~nm}, 16$ $\mathrm{mm}$, and $71 \mathrm{~mW}$ at $65.95 \mathrm{kHz}$ repetition rate, respectively. From these results, the FDML technique in the swept laser source has proved to be an effective method for enhancing the overall performance of the swept laser.

\section{Fiber Probes for Optical Coherence Tomography Imaging}

\subsection{Design of fiber probes}

Fiber-based OCT system has capability of using an optic fiber probe. The light beam usually is guided through a SMF and is focused on a tested sample by an optical lens attached to the SMF. Back-reflected light carrying information about the sample is coupled back into the fiber, and then signal detection and data processing systems will collect the useful information for imaging. The optic probe is one of the crucial parts of OCT imaging system. The beam profile, i.e. working distance (focal distance from lens surface), depth of field (two times the Raleigh range), and spot size (waist diameter) of the optical probe in the sample 
will directly determine the properties of the image such as image location, depth, and resolution. Thus, image quality and light coupling efficiency from the sample will be directly influenced by the beam quality of the probe. For the best optical performance of a probing lens, its beam profile must be designed to be consistent with the light penetration depth in the sample. In most biomedical imaging systems, light from the probe will be directed into a turbid tissue. Based on interaction properties of light with turbid tissue (Sainter et al., 2002) the range of penetration depth is from $0.5-3 \mathrm{~mm}$ at near infrared wavelengths. For example, the penetration depths are $0.7 \mathrm{~mm}$ and $3.0 \mathrm{~mm}$ in human skin and liver respectively at $1300 \mathrm{~nm}$, a conventional wavelength used in OCT systems. Thus, for designing an optical probe, working distance should be in the range of $0.4-1.2 \mathrm{~mm}$ in the air that depends on the tissues to be tested. There is a tradeoff between the depth of field and beam spot size because the depth of field of a lens is positively related to the square of the spot size according to the theory of Gaussian beam. A large depth of field unavoidable results in a large spot size. Thus, the optimal depth of field is in the range of $0.8-1.5 \mathrm{~mm}$ in the air; this keeps the spot size in the range of $26-35 \mu \mathrm{m}$ at the $1300 \mathrm{~nm}$ wavelength. For an ultra-small optical lens, it is not possible to achieve a large working distance by directly attaching a lens to a SMF because of the strong focus ability of lens and the small mode field diameter (MFD) of the SMF. A fiber spacer with a homogeneous index of refraction has to be added between the SMF and the lens for beam expansion prior to focusing to obtain a larger working distance. Therefore, theoretical modeling becomes necessary to obtain a fiber lens with optimized optical beam performance for imaging different tissues.

In this section, we study two types of fiber lenses, GRIN and ball fiber lenses. We used ZEMAX to design both GRIN and ball fiber lenses by choosing an appropriate surface type and analysis method.

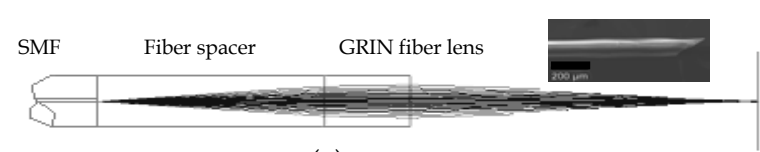

(a)

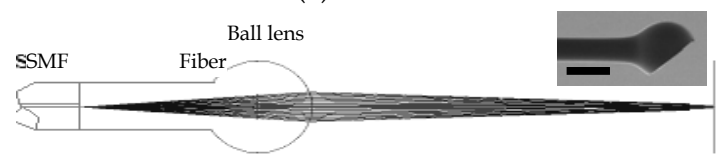

(b)

Fig. 12. ZEMAX layouts of ray trace for the GRIN (a) and ball (b) fiber lens systems, respectively. Insets: scanning electron micrographs of the GRIN and ball fiber lens tips fused with angle-polished beam director, respectively. Marks are $200 \mu \mathrm{m}$.

For a GRIN fiber lens, the gradient surface type used in ZEMAX will depend on the profile of the refractive index. The refractive index profile of the GRIN fiber lens used in this work is a radial index gradient, which is very similar to that of a conventional glass GRIN (or called SELFOC) lens. The index of refraction is highest in the center of the lens and decreases with radial distance from the axis. The following quadratic equation closely describes the refractive index of a GRIN fiber lens (Kogelnik \& Li, 1966; Emkey \& Jack, 1987): 


$$
n(r)=n_{0}\left(1-\frac{g^{2}}{2} r^{2}\right)
$$

where $r$ is the radial position from the axis, $n_{0}$ is refractive index on the lens axis, $g$ is the gradient constant. The pitch, $p=2 \pi / g$, is the spatial period of the ray trajectory. For modeling of the ball fiber, the Standard Surface type in ZEMAX is employed. Fig. 12 (a) and (b) show the ZEMAX ray trace layout of the GRIN and ball fiber lenses, respectively, with their scanning electron micrographs in the insets. The working distance, depth of field and spot size were calculated in ZEMAX using the Gaussian beam theory. The theoretical results will be shown and discussed in comparison with the experimental results below.

\subsection{Fabrication Method of the fiber probes}

The GRIN and ball fiber lens probes were made from a standard Corning SMF-28 single mode fiber as the principal light guide, a fiber spacer and a GRIN or a ball fiber lens as the focusing lens. For the GRIN lens probe, a fiber spacer with same outer diameter $(0.125 \mathrm{~mm})$ as SMF-28 was fusion-spliced via arc welds to the Corning SMF-28 and then accurately cleaved to a theoretically-determined length. A GRIN fiber was then fusion-spliced to the cleaved fiber spacer and precisely cleaved at a pre-calculated length to generate a desired beam-distance profile (i.e., working distance, depth of field, and spot size). For a short working distance probe, the section of the fiber spacer was omitted resulting in a simple fabrication process. For the ball lens probe, a fiber spacer was fusion-spliced via arc welds to the Corning SMF-28 and then accurately cleaved to a theoretically-determined length plus extra $0.2 \mathrm{~mm}$. The tip of the fiber spacer then was fused via arc welds to a perfect ball shape by inputting an appropriate fusion setting. To ensure minimum back-reflection for both probes, the indexes of the fiber spacer and the center of GRIN fiber were matched to the core index of the SMF.

We used a conventional low cost off-the-shelf optical multi-mode GRIN fibers as the GRIN lens, which has $0.1 \mathrm{~mm}$ core size, $0.14 \mathrm{~mm}$ outer diameter, a core refractive index $n_{0}=1.487$, and a gradient constant $g=3.76$ at $1300 \mathrm{~nm}$ (Prime Optical Fiber Corp., Taiwan). The fiber spacers (Prime Optical Fiber Corp., Taiwan) are made of pure silica without a core. Fusionsplicing was processed using an Ericsson FSU 995 fusion-splicer and an EFC11 fiber cleaver (3SAE Technologies, TN, USA). The spliced interfaces produced minimum back-reflections since the mechanical strength at the interface was similar to that of the untreated fiber. The desired focused beam profile was obtained by tailoring the length of the fiber spacer and parameters of fiber lenses (length of GRIN fiber and diameter of the ball) based on the theoretical results. We fabricated the different variations of the GRIN and ball fiber lens modules with the different length of the fiber spacer and the different lens parameter. All samples were listed in Table 3 with detailed information.

A beam profile measurement system (BeamView Analyzer, OR, USA) with an infrared camera (Electrophysics, NJ, USA) and a Super Luminous Diode source (Covega, MD, USA) with $60 \mathrm{~nm} 3 \mathrm{~dB}$ bandwidth at $1310 \mathrm{~nm}$ center wavelength was used to characterize the beam parameters of the lens system. A 40X JIS microscopic objective lens and a related objective tube were attached to the input window of the camera to increase the image resolution. The horizontal and vertical resolutions of the system were $1.0 \mu \mathrm{m}$ and $1.1 \mu \mathrm{m}$, respectively. The 
distribution of light intensity at various distances along the direction of propagation after the lens was accurately measured by the beam profile system. Working distance, depth of focus, $1 / \mathrm{e}^{2}$ spot size, and Gaussian fitting were analyzed from the measured intensity distribution. The results demonstrated in this work are all in the air medium.

In addition, after characterization of the lens, a beam director could be attached to the lens for a side-view probe. The different attaching methods were used for the two lenses. For the GRIN lens, a fiber spacer was fusion-spliced to the finished lens end as a beam director by polishing the end of the fiber spacer to a 45 degrees angle and coating the polished surface with a total reflection film. This then allowed the beam to be reflected at a 90 degrees angle creating a side-view probe. For the ball lens probe, the beam can be 90-110 degree reflected by a 45-50 degree polished face on the ball lens by the total internal reflection. Insets of Fig. 12 (a) and (b) show the typical scan electron microscope (SEM) pictures of the GRIN and ball fiber lens tip fused with beam directors, respectively. The fiber lens tip together with a tubing system and a connected linearly scanning or 360 degrees rotated motor could be built as an endoscope, or catheter, or a needle probes. The diameter of these probes could be as small as $0.4 \mathrm{~mm}$ which is best suitable for internal in situ and in vivo biomedical imaging, diagnostic, guided surgery, and treatment with a minimal invasion.

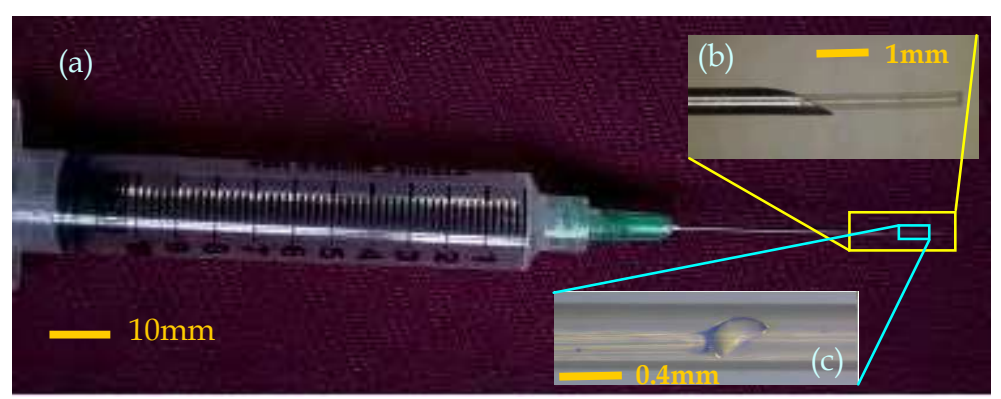

Fig. 13. OCT side-view needle probe showing the tubing and angle-polished ball lens. (a) needle probe. (b) retracted needle tip with protective tubing. (c) protective tubing and exposing the lens.

As a sample, a needle probe designed for the OCT imaging in this work is shown in Fig 13 (a). The lens and the uncoated portion of the SMF are protected in a transparent inner catheter (OD $0.49 \mathrm{~mm}$ ) shown in Fig. $13(\mathrm{~b}, \mathrm{c})$. The buffered portion of the fiber is attached to an outer flexible catheter after the syringe (OD $1.4 \mathrm{~mm}$ ), which is fastened onto a modified syringe piston, while the transparent inner catheter is inserted into a $21 \mathrm{G}$ (OD $0.81 \mathrm{~mm}$ ) echogenic spinal needle (VWR, Mississauga, ON, Canada). After insertion into the tissue, the needle can be drawn back while the optical probe stays inside of the tissue as shown in Fig. 13 (b). The probe is then scanned axially inside the tissue driven by a linear scanner, such that a two dimensional OCT image is formed. If a fiber GRIN lens is used, the size of the inner catheter could be as small as $0.4 \mathrm{~mm}$ because the diameter of the GRIN lens is smaller than the fiber ball lens. 


\begin{tabular}{|c|c|c|c|c|c|c|}
\hline \multirow{2}{*}{ Sp.\# } & $\begin{array}{c}\text { Length of } \\
\text { Fiber } \\
\text { Spacer } \\
\end{array}$ & \multicolumn{2}{|c|}{ Fiber Lens } & \multicolumn{2}{c|}{ Measured Beam Properties } \\
\cline { 3 - 7 } & $(\mathrm{mm})$ & $\begin{array}{c}\text { Type/Diameter } \\
(\mu \mathrm{m})\end{array}$ & $\begin{array}{c}\text { Length } \\
(\mathrm{mm})\end{array}$ & $\begin{array}{c}\text { Working } \\
\text { Distance } \\
(\mathrm{mm})\end{array}$ & $\begin{array}{c}\text { Depth of } \\
\text { Field } \\
(\mathrm{mm})\end{array}$ & $\begin{array}{c}\text { Spot } \\
\text { Size } \\
(\mu \mathrm{m})\end{array}$ \\
\hline 1 & 0 & GRIN100/140 & 0.60 & 0.18 & 0.16 & 13 \\
\hline 2 & 0 & GRIN100/140 & 0.55 & 0.20 & 0.30 & 16 \\
\hline 3 & 0 & GRIN100/140 & 0.52 & 0.28 & 0.50 & 22 \\
\hline 4 & 0 & GRIN100/140 & 0.50 & 0.38 & 0.60 & 23 \\
\hline 5 & 0 & GRIN100/140 & 0.48 & 0.41 & 0.85 & 25 \\
\hline 6 & 0 & GRIN100/140 & 0.46 & 0.40 & 1.30 & 30 \\
\hline 7 & 0 & GRIN100/140 & 0.45 & 0.38 & 1.45 & 32 \\
\hline 8 & 0.48 & GRIN100/140 & 0.17 & 1.00 & 0.95 & 28 \\
\hline 9 & 0.48 & GRIN100/140 & 0.16 & 1.10 & 1.5 & 35 \\
\hline 10 & 0.48 & GRIN100/140 & 0.145 & 1.20 & 1.8 & 41 \\
\hline 11 & 0.48 & GRIN100/140 & 0.14 & 1.05 & 2.0 & 45 \\
\hline 12 & 0.52 & Ball Lens/300 & $\sim$ & 1.00 & 3.6 & 50 \\
\hline 13 & 0.55 & Ball Lens/300 & $\sim$ & 1.4 & 2.1 & 45 \\
\hline 14 & 0.62 & Ball Lens/300 & $\sim$ & 1.20 & 1.1 & 27 \\
\hline 15 & 0.70 & Ball Lens/300 & $\sim$ & 1.00 & 0.5 & 20 \\
\hline 16 & 0.74 & Ball Lens/300 & $\sim$ & 0.90 & 0.33 & 16 \\
\hline
\end{tabular}

Table 3. Structures of the various fiber lenses with measured beam properties.

\subsection{Characterization Results of the fiber probes}

The measured results of the beam properties are listed in Table 3 along with detailed descriptions of the listed samples. For each sample, optical intensity distribution data on the radial (i.e. $x$ and $y$ ) planes were collected along the beam propagation (i.e. optical axial $z$ ) direction from the plane of the first half peak intensity (begin-plane), through the maximum intensity plane, i.e. focus plane (center-plane), to the second half peak intensity plane (endplane). Beam properties including working distance, spot size, and depth of field were analyzed by measured intensity distribution data with distance from the lens surface to the focal plane, $1 / \mathrm{e}^{2}$ beam diameter at the focal plane, and the distance between the begin-plane and the end-plane, respectively. The theoretical and experimental results of working distance, depth of focus, and spot size of different variations vs. length of GRIN fiber or diameter of the ball lens (bottom x-axis), and length of fiber spacer (top x-axis) are shown in Fig. $14(\mathrm{a}),(\mathrm{b})$, and (c), respectively, where, lines represent the theoretical results from ZEMAX at $1300 \mathrm{~nm}$, amount them, dark doted line represent GRIN fiber lens without a fiber spacer, dark and light solid lines represent GRIN fiber lens with a constant length of fiber spacer $(0.48 \mathrm{~mm})$ and a constant length of GRIN fiber $(0.17 \mathrm{~mm})$, respectively; dark and light dash lines represent ball fiber lens with a constant length of fiber spacer $(0.62 \mathrm{~mm})$ and a constant diameter of the ball $(0.30 \mathrm{~mm})$, respectively. The experimental results were represented by points, amount them, triangle points represent GRIN fiber lens and square points represent the ball lens.

From the theoretical result shown in Fig. 14, short working distance $(<0.4 \mathrm{~mm})$ could be obtained by the GRIN fiber lens without fiber spacer shown as the dark dotted lines. To 
obtain larger working distance, a fiber spacer has to be used. In these cases, the working distance varies sharply with the length of GRIN fiber for the GRIN fiber lens and with the diameter of the ball for the ball fiber lens, but it varies less sharply with the length of the fiber spacer. The working distances have saturated values for each case. By compensating the working distance with the depth of field and the spot size, the optimized parameters (i.e. $0.9-1.2 \mathrm{~mm}$ working distance, $0.9-1.1 \mathrm{~mm}$ depth of filed, and $<30 \mu \mathrm{m}$ spot size) are not at the position of the largest working distance, instead, the optimized positions are around 0.17 $\mathrm{mm}$ length of the GRIN fiber with $0.48 \mathrm{~mm}$ length of fiber spacer for GRIN fiber lens and 0.3 $\mathrm{mm}$ diameter of ball and $0.62 \mathrm{~mm}$ length of fiber spacer for ball fiber lens. The results of the experimental results as shown in Fig. 14 and Table 3 indicated that the ZEMAX numerical optical design software were in a good agreement with the experimental results. We obtained the working distance of $1.0 \mathrm{~mm}$, the depth of field of $0.95 \mathrm{~mm}$, and the spot size of $28 \mu \mathrm{m}$ from a GRIN fiber lens module (sample \#8) and the working distance of $1.2 \mathrm{~mm}$, the depth of field of $1.1 \mathrm{~mm}$, and the spot size of $27 \mu \mathrm{m}$ from a ball fiber lens module (sample $\# 14)$.

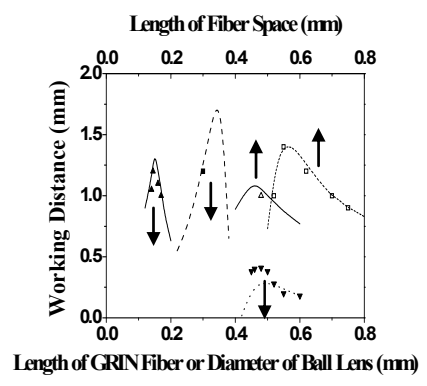

(a)

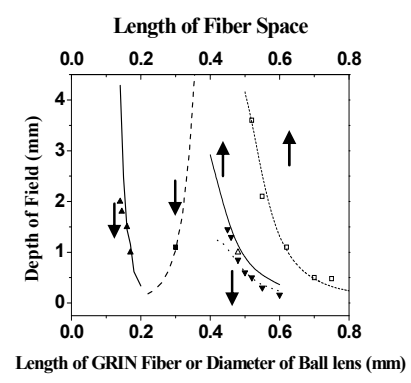

(b)

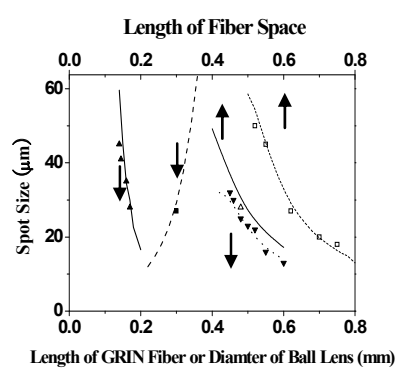

(c)

Fig. 14. Theoretical and experimental results of working distance (a), depth of field (b), and spot size (c) vs. length of GRIN fiber or diameter of the ball lens (bottom $x$ axis), and length of fiber spacer (top $x$ axis), where, lines represent the theoretical results from ZEMAX at $1300 \mathrm{~nm}$, amount them, dark doted line represent GRIN fiber lens without a fiber spacer, dark and light solid lines represent GRIN fiber lens with a constant length of fiber spacer $(0.48 \mathrm{~mm})$ and a constant length of GRIN fiber $(0.17 \mathrm{~mm})$, respectively; dark and light dash lines represent ball fiber lens with a constant length of fiber spacer $(0.62 \mathrm{~mm})$ and a constant diameter of the ball $(0.30 \mathrm{~mm})$, respectively. The related experimental results were represented by the points.

We found quality of the beam depends very much on the quality of the surface cleaving and the alignment of the fusion-splicing between the fiber spacer and the fiber lens. The high quality beam is easier to obtain for the probe with the ball fiber lens because the ball is made from the fiber spacer and there is no interface between the fiber spacer and the ball lens. By well controlling the cleaving and the fusion-splicing, we obtained high quality of beam for the probe of the GRIN fiber lens as well. Fig. 15 shows measured and Gaussian-fitted 1/e ${ }^{2}$ intensity beam diameters along the axial distance $z$ (zero is the position of the lens surface) at $x$ (horizontal) and $y$ (vertical) radial coordination in the distance range of depth of field for the samples \#1, \#3 and \#8 with the GRIN fiber lenses as shown in Table 3. 


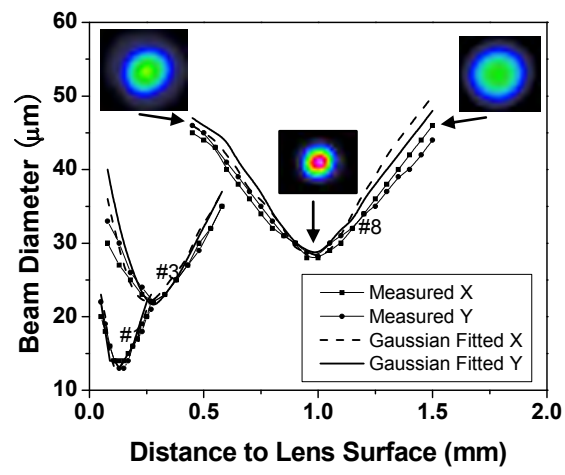

Fig. 15. Measured and Gaussian-fitted $1 / \mathrm{e}^{2}$ intensity beam diameters along the axial distance (zero is the position of the lens surface) at $x$ (horizontal) and $y$ (vertical) radial coordination in the distance range of depth of field of the samples \#1, \#3 and \#8, which was made from the GRIN fiber lens. Insets: the cross-section beam profiles at three planes for the sample \#8.

For each curve in Fig. 15, the smallest beam diameter value presents the spot size, $x-$ coordinate value at the pole point indicates the working distance, and the distance range of the curve indicates the depth of field. The working distance of $0.18 \mathrm{~mm}$, depth of field of $0.16 \mathrm{~mm}$, and spot size of $13 \mu \mathrm{m}$ were obtained for the sample \#1 with $0.60 \mathrm{~mm}$ length of 100/140 GRIN fiber lens which was directly attached to SMF without fiber spacer. If the length of GRIN fiber was reduced to $0.52 \mathrm{~mm}$, the beam became less converged resulting the three parameters increased slightly to $0.28 \mathrm{~mm}, 0.5 \mathrm{~mm}$, and $22 \mu \mathrm{m}$, respectively. When a $0.48 \mathrm{~mm}$ fiber spacer was inserted between SMF and GRIN fiber and the length of GRIN fiber is reduced to $0.17 \mathrm{~mm}$, significant less converged beam is observed. The working distance of $1.0 \mathrm{~mm}$, depth of field of $0.95 \mathrm{~mm}$, and spot size of $28 \mu \mathrm{m}$ were obtained for sample \#8.

The measured beam diameters are well matched to Gaussian-fitted values in the center (focused) regions, but have small deviations in the two edge side regions as shown in Fig. 15. The insets in Fig. 15 shown images of the cross-section beam profile at begin, center, and end planes, respectively, for the sample \#8. The circular shapes of the profile images indicate high $\mathrm{x}$ and $\mathrm{y}$ symmetry of the beam profiles through all the range of depth of filed.

Considering chromatic aberrations, from ZEMAX simulation for the ball fiber lens in the wavelength range of $1260-1370 \mathrm{~nm}$, the relative variations of the working distance, depth of field and spot size were calculated all smaller than $4.0 \%$. For the GRIN fiber lens, the range of the zero-dispersion wavelengths, $\left\{\lambda_{0}\right\}$ is $1297-1316 \mathrm{~nm}$. The zero-dispersion slope, $\mathrm{S}_{0}$ equal to or smaller than $0.101 \mathrm{ps} / \mathrm{nm}^{2}-\mathrm{km}$. Using the standard formula of fiber dispersion, $D(\lambda)=S_{0}\left[\lambda-\lambda_{0}{ }^{4} / \lambda^{3}\right] / 4(\mathrm{ps} / \mathrm{nm}-\mathrm{km})$, we calculated the changes of refractive index in the 1260 - $1370 \mathrm{~nm}$ wavelength range. By using these values in ZEMAX, we calculated the 
relative changes of the working distance, depth of field and spot size were all smaller than $3 \%$. Based on our results, the desired beam profile for the application of optical biomedical imaging systems can be obtained by the GRIN and ball fiber lens with or without fiber spacers. The technique described here possesses a high degree of flexibility for designing ultra-small optical probes with different beam shapes for the different tissue imaging.

\section{OCT Imaging}

Fig. 16 (a) and (d) show SS-OCT images of human finger in vivo taken using fiber probes \# 14 (working distance, depth of field and spot diameter of $1.2 \mathrm{~mm} 1.1 \mathrm{~mm}$, and $27 \mu \mathrm{m}$ ) and \# 16 (working distance, depth of field and spot diameter of $0.9 \mathrm{~mm} 0.33 \mathrm{~mm}$, and $16 \mu \mathrm{m}$ ) acquired by our catheter-based complex SS-OCT using our 3x3 Mach-Zehnder interferometer with unbalanced differential detection technique with image size of $5 \times 2 \mathrm{~mm}$. The image depth shown in Fig. 16 (a) is slightly larger than that in Fig. 16 (b), but the image is blurrier in Fig. 16 (a) than that in Fig. 16 (b), which taken by the probe with larger depth of field and spot size. The image shown in Fig. 16 (b) has higher resolution than that in Fig. 16 (a), which can be seen clearly with finer structures in layer of epidermis (grey arrow), sweat gland (white arrow), and blood vessel in subcutis layer (black arrow).

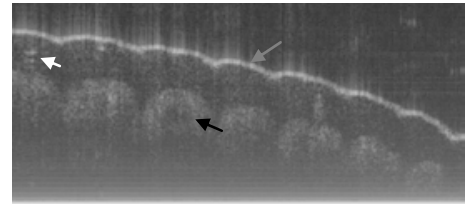

(a)

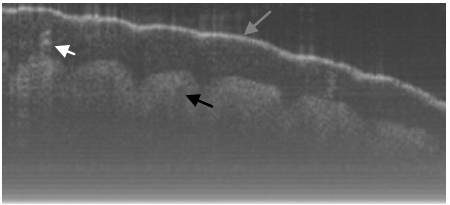

(b)

Fig. 16. In vivo human finger SS-OCT images taken with probe \#14 (working distance, depth of field and spot diameter of $1.2 \mathrm{~mm} 1.1 \mathrm{~mm}$, and $27 \mu \mathrm{m}$ ) and \# 16 (working distance, depth of field and spot diameter of $0.9 \mathrm{~mm} 0.33 \mathrm{~mm}$, and $16 \mu \mathrm{m}$ ) acquired by our catheter-based complex SS-OCT using our $3 \times 3$ Mach-Zehnder interferometer with unbalanced differential detection technique with image size of $5 \times 2 \mathrm{~mm}$.

Fig. 17 shows ex vivo images of coronary artery of rabbit with forward-view $(a, b)$ probe with ball lens \#16 and side-view (c, d) probe with GRIN lens \#5 acquired by our catheter-based complex SS-OCT using our 3x3 Mach-Zehnder interferometer with unbalanced differential detection technique with image size of $2.5 \times 2 \mathrm{~mm}$ by scanning the probe along the artery $(\mathrm{a}, \mathrm{c})$ and scanning cross the artery $(b, d)$. Three layers of tunica intima, tunica media, and tunica adventitia for the coronary artery are viewed clearly as indicated by the gray, black, and white arrows in all four images in Fig. 17. The fine layers of muscle and elastic fiber in the tunica media of the coronary artery are shown obviously in the images obtained by scanning the probe cross the artery. 

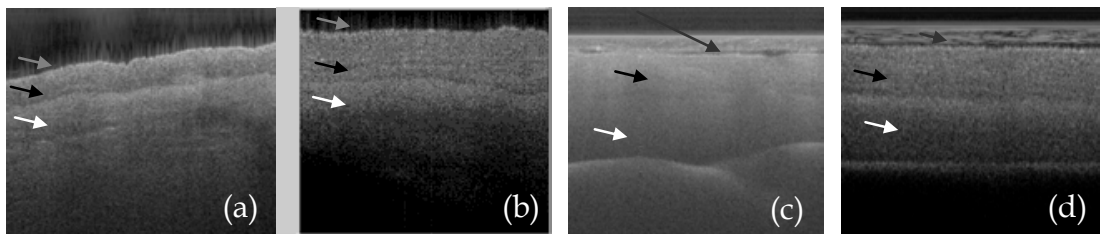

Fig. 17. Ex vivo images of coronary artery of rabbit scanning along the artery $(\mathrm{a}, \mathrm{c})$ and scanning cross the artery $(b, d)$ with forward-view probe $(a, b)$ with ball lens \#16 and sideview (c, d) probe with GRIN lens \#5 acquired by our catheter-based complex SS-OCT using our 3x3 Mach-Zehnder interferometer with unbalanced differential detection technique.

Fig. 18 shows ex vivo images of heart atrium $(a, b)$, artery (c) of mice, and heart atrium (d, e, f) of pig with forward-view probe with ball lens \#16 acquired by our catheter-based complex SS-OCT using our $3 \times 3$ Mach-Zehnder interferometer with unbalanced differential detection technique with image size of $2 \times 2 \mathrm{~mm}$. The shapes and structures of these internal organs of small and large animals are viewed clearly from these OCT images.

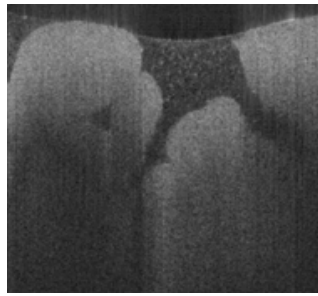

(a)

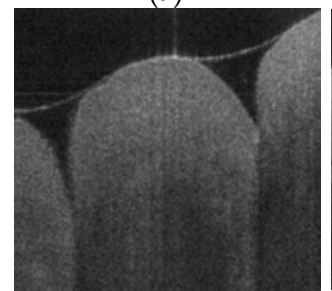

(d)

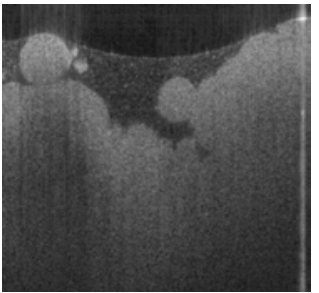

(b)

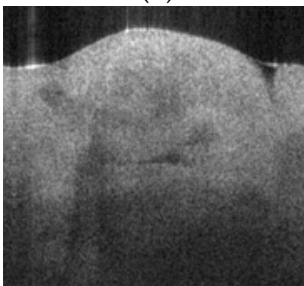

(e)

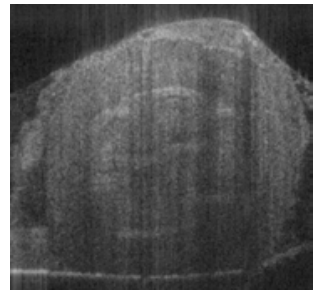

(c)

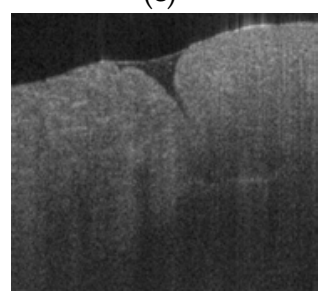

(f)

Fig. 18. Ex vivo images of heart atrium $(\mathrm{a}, \mathrm{b})$, artery (c) of mice, and heart atrium $(\mathrm{d}, \mathrm{e}, \mathrm{f})$ of pig acquired by our catheter-based complex SS-OCT using our $3 \times 3$ Mach-Zehnder interferometer with unbalanced differential detection technique.

The in vivo and ex vivo OCT images shown in Fig. 16, 17, and 18 indicate that our catheterbased complex SS-OCT system is capable of imaging the biomedical tissues or the inside organs for human, small and big animals and it is most suitable for applications of diagnosis and guided surgery. 


\section{Acknowledgments}

Authors gratefully thank Dan P. Popescu and Michael G. Sowa from Institute for Biodiagnostics of National Research Council of Canada, and Tim Cheung from Heart Institute of University of Ottawa for their providing of the animal tissues.

\section{Conclusion}

We have demonstrated a catheter-based full range swept-source optical coherence tomography system. A $3 \times 3$ quadrature Mach-Zehnder interferometer with a new unbalanced differential detection method for SS-OCT have been firstly presented. Using this setup, a complex conjugate artifact suppression of $27 \mathrm{~dB}$ has been achieved. A 90\% phase shift between the two interferometric outputs was obtained thereby eliminating the need for further trigonometric calculations. Also, our setup resulted in a $4 \mathrm{~dB}$ increase in the signalto-noise ratio compared to a similar interferometer with the commonly used balanced detection technique. We have then demonstrated a high-performance wavelength-swept laser that uses a high-efficiency semiconductor optical amplifier, a high-speed polygonbased narrowband scanning filter, and a Fourier domain mode lock technique. This laser produced $71 \mathrm{~mW}$ average output power with an instantaneous linewidth of $0.09 \mathrm{~nm}$, and it could be tuned over a wavelength range of $113 \mathrm{~nm}$ at a repetition rate of $65.95 \mathrm{kHz}$. We also constructed an OCT system that uses our laser source where we have shown that its parameters are optimized for this application. We presented next the design, construction and beam profile characterization of different variations of graded-index and ball fiber lenses, which were recently proposed for ultra-small probes for OCT imaging. Those fiber lens modules were made of single mode fibers and GRIN and ball fiber lenses with/without fiber spacers between them. We used fusion-splicing in between the fibers, lenses and spacers to ensure high quality light transmission. We found that beam-distance profiles (i.e. $0.4-1.2 \mathrm{~mm}$ of focus distance, $0.8-1.5 \mathrm{~mm}$ of depth of field, and $26-35 \mu \mathrm{m}$ of spot size) can be obtained by precisely adjusting the lengths of the fiber spacer and the GRIN fiber lens or diameter of the ball lens for the different tissue imaging in human beings and animals. We obtained very high quality focused Gaussian beam profiles with high $\mathrm{x}$ and $\mathrm{y}$ symmetry using the conventional multi-mode GRIN fibers and home-made fiber ball lenses. Their high quality beam and ultra-small size make such fiber lens based probes very valuable for biomedical optical imaging systems. The in vivo and ex vivo OCT images acquired by our catheter-based full range SS-OCT system indicate that this system is capable of imaging the biomedical tissues or inside organs for human, small/big animals and it is most suitable for applications of diagnosis and guided surgery.

\section{References}

Bilenca, A. et al. (2006). Numerical study of wavelength-swept semiconductor ring lasers: the role of refractive-index nonlinearities in semiconductor optical amplifier and implications for biomedical imaging application, Opt. Lett. 31, 760-762, ISSN: 01469592.

Chinn, S.R.; Swanson, E. A. \& Fujimoto, J. E. (1997). Optical coherence tomography using a frequency-tunable optical source, Opt. Lett., 22, 340-342, ISSN: 0146-9592. 
aChoma, M.A. et al. (2003). Sensitivity advantage of swept source and Fourier domain optical coherence tomography, Opt. Exp. 11, 2183-2189, ISSN: 1094-4087.

bChoma, M.A.; Yang, C. \& Izatt, J.A. (2003). Instantaneous quadrature low-coherence interferometry with $3 \times 3$ fiber-optic couplers, Opt. Lett. 28, 9672162-2164, ISSN: 01469592.

De Boer, J.F. et al. (2003). Improved signal-to-noise ratio in spectral-domain comared with time-domain optical coherence tomography, Opt. Lett. 28, 2067-2069, ISSN: 01469592.

Diaz-Sandoval, L. J. (2005). Optical Coherence Tomography as a tool for percutaneous coronary interventions, Catheter. And Cardio. Interventions, 65, 492-496, ISSN: 15221946.

Emkey W. L. \& Jack, C. A. Analysis and evaluation of graded-index fiber-lenses, J. of Lightwave Tech., LT-5, 1156-1164, ISSN:0733-8724.

Fercher, F. et al. (1995). Measurement of intraocular distances by backscattering spectral interferometry, Opt. Commun. 117, 443-448, ISSN: 0030-4018.

Fujimoto, J. G. et al. (1999) High resolution in vivo intra-arterial imaging with optical coherence tomography, Heart, 82, 128-133,

Hausler, G. \& Lindner, M. W. (1998). Coherence Radar and Spectral Radar - new tools for dermatological diagnosis, J. Biomed. Opt. 3, 21-31, ISSN: 1083-3668.

Hecht, E. (1997) Opics (Addison-Wesley, Reading, Mass.).

Huang, D. et al. (1991). Optical coherence tomography, Science, 254, 1178-1181, ISSN: 00368075.

Huber, R. et al. (2005). Amplified, frequency swept lasers for frequency domain reflectometry and OCT imaging: design and scaling principles, Opt. Exp., 13, 35133528, ISSN: 1094-4087.

Huber, R.; Adler, D.C. \& Fujimoto, J.G. (2006). Buffered Fourier domain mode locking:unidirectional swept laser sources for optical coherence tomography imaging at 370,000 lines/s, Opt. Lett. 31, 2975-2977, ISSN: 0146-9592.

Huber, R.; Wojtkowski, M. \& Fujimoto, J.G. (2006) Fourier domain mode locking (FDML): a new laser operating regime and applications for optical coherence tomography, Opt. Exp. 14, 3225-3237, ISSN: 1094-4087.

Jafri, M. S. et al. (2005) Optical coherence tomography in the diagnosis and treatment of neurological disorders, J. Biomed. Opt. 10(5) 051603, ISSN: 1083-3668.

Kogelnik, H. W. \& Li, T. (1966) Laser Beam and Resonators, Appl. Opt. 5, 1550, ISSN: 00036935.

Leitgeb, R.; Hitzenberger, C.K. \& Fercher, A.F. (2003). Performance of Fourier domain vs. time domain optical coherence tomography, Opt. Exp. 11, 889-894, ISSN: 1094-4087.

$\mathrm{Li}, \mathrm{H}$. et al. (2006). Feasibility of interstitial doppler optical coherence tomography for in vivo detection of microvascular changes during photodynamic therapy, Lasers in Surgery and Medicine 38, 754-761, ISSN:0196-8092.

$\mathrm{Li}$, X. et al. (2000). Imaging needle for optical coherence tomography, Opt. Lett. 25, 15201522, ISSN: 0146-9592.

Mao, Y. et al. (2007) Graded-index fiber lens proposed for ultrasmall probes used in biomedical imaging, App. Opt., 46, 5887-5894, ISSN: 0003-6935. 
aMao, Y. et al. (2008). 3x3 Mach-Zehnder Interferometer with Unbalanced Differential Detection for Full Range Swept-Source Optical Coherence Tomography, Appl. Opt. 47, ISSN:2004-2010.

bMao, Y. et al. (2008) Design and Implementation of Fiber Lenses for Ultra-Small Probes Used in Biomedical Imaging, Proc. SPIE, 6826, 68261A, ISSN: 0277-786X.

Mao, Y. et al. (2009). High Performance Wavelength-Swept Laser with Mode-Locking Technique for Optical Coherence Tomography, Opt. Comm., 282, 88-92, ISSN:00304018.

Oh, W. Y. et al. (2005). $115 \mathrm{kHz}$ tuning repetition rate ultrahigh-speed wavelength-swept semiconductor laser, Opt. Lett. 30, 3159-3161, ISSN: 0146-9592.

Oh, W. Y. (2008). Speed polarization sensitive optical frequency domain imaging with frequency muliplexing, Opt. Exp. 16, 1096-1103, ISSN: 1094-4087.

Podoleanu, A. Gh. (2000). Unbalanced versus balanced operation in an optical coherence tomography system, Appl. Opt. 39, 173-182, ISSN:2004-2010.

Priest, R. G. (1982) Analysis of fiber interferometer utilizing 3x3 fiber coupler, IEEE Trans. Microwave Theory Tech, MTT-30, 1589-1591, ISSN:0018-9480.

Rao, B. et al. (2007). Investigation of coherence amplification with a semiconductor optical amplifier employed in a swept source OCT system, Proc. of SPIE, 6429, 642924-1, ISSN: 0277-786X.

Reed, W. A.; Yan, M. F. \& Schnitzer, M. J. (2002). Gradient-index fiber-optic microprobes for minimally invasive in vivo low-coherence interferometry, Opt. Lett. 27, 1794-1796, ISSN: 0146-9592.

Rollins, A. M. \& Izatt, J. A. (1999). Optimal interferometer designs for optical coherence tomography, Opt. Let., 24, 1484-1486, ISSN: 0146-9592.

Sainter, A. W.; King, T. A. \& Dickinson,V Theoretical comparison of light sources for use in optical coherence tomography, Proc. SPIE 4619, 289-299, ISSN: 0277-786X.

Sarunic, M.V. et al. (2005). Instantaneous complex conjugate resolved spectral domain and swept-source OCT using 3x3 fiber couplers, Opt. Exp. 13, 957-967, ISSN:1094-4087.

Sarunic, M.V.; Applegate, B.E. \& Izatt, J.A. (2006). Real-time quadrature projection complex conjugate resolved Fourier domain optical coherence tomography, Opt. Lett. 31, 2426-2428, ISSN: 0146-9592.

Sheem, S. K. (1981) Optical fiber interferometers with [3x3] directional couplers: analysis, J. Appl. Phys. 52, 3865-3872, ISSN:0021-8979.

Shishkov, M.; Bouma, B. E. \& Tearney, G. J. (2006). System and method for optical coherence tomography, U.S. Patent 20060067620A1 (Mar. 30).

Sticker, M. et al. (2001). Quantitative differential phase measurement and imaging in transparent and turbid media by optical coherence tomography, Opt. Lett. 26, 114116, ISSN: 0146-9592.

Swanson, E. (2002). Ultra-small optical probes, imaging optics, and methods for using same, U.S. Patent 6445939 (Sept. 3).

Takada, K. et al. (1987). New measurement system for fault location in optical waveguide devices based on an interferometric technique, App. Opt. 26, 1603-1606, ISSN: 00036935.

Tran, P. H. et al. (2004). In vivo endoscopic optical coherence tomography by use of a rotational microelectromechanical system probe, Opt. Lett. 29, 1236-1238, ISSN: 0146-9592. 
Vakoc, B.J.et al. (2006) Elimination of depth degeneracy in optical frequency_domain imaging through polarization-based optical demodulation, Opt. Lett. 31, 362-364, ISSN: 0146-9592.

White, B. R. et al. (2003). In vivo dynamic human retinal blood flow imaging using ultrahigh-speed spectral domain optical coherence tomography, Opt. Exp. 11, 3490-3497, ISSN: 1094-4087.

Yang, V. X. D. et al. (2005). Endoscopic Doppler optical coherence tomography in human gastrointestinal tract: initial experience, Gastrointest. Endosc. 61, 879-890, ISSN: 00165085.

Yang, V. X. D. et al. (2005). Interstitial Doppler optical coherence tomography, Opt. Lett. 30, 1791-1793, ISSN: 0146-9592.

Yazdanfar, S.; Rollins, A. M. \& Izatt, J. A. (2000) Imaging and velocimetry of the human retinal circulation with color Doppler optical coherence tomography, Opt. Lett. 25, 1448-1450, ISSN: 0146-9592.

Youngquist, R. C.; Carr, S. \& Davies, D. E. N. (1987). Optical coherence-domain reflectometry: A new optical evaluation technique, Opt. Lett., 12, 158-160, ISSN: 0146-9592.

aYun, S.H. et al. (2003). High-speed optical frequency-domain imaging, Opt. Exp. 11, 29532963, ISSN: 1094-4087.

bYun, S. H. et al. (2003). High-speed wavelength-swept semiconductor laser with a polygonscaner-based wavelength filter, Opt. Lett. 28, 1981-1983, ISSN: 0146-9592.

Yun, S.H. et al. (2004). Removing the depth-degeneracy in optical frequency domain imaging with frequency shifting, Opt. Exp. 12, 4822-4828, ISSN: 1094-4087.

Zhang, J. et al. (2004). Full range polarization-sensitive fourier domain optical coherence tomography, Opt. Exp. 12, 6033-6039, ISSN: 1094-4087.

aZhao, Y. et al. (2000). Phase-resolved optical coherence tomography and optical Doppler tomography for imaging blood flow in human skin with fast scanning speed and high velocity sensitivity, Opt. Lett. 25, 114-116, ISSN: 0146-9592.

bZhao, Y. et al. (2000). Doppler standard deviation imaging for clinical monitoring of in vivo human skin blood flow, Opt. Lett. 25, 1358-1360, ISSN: 0146-9592. 


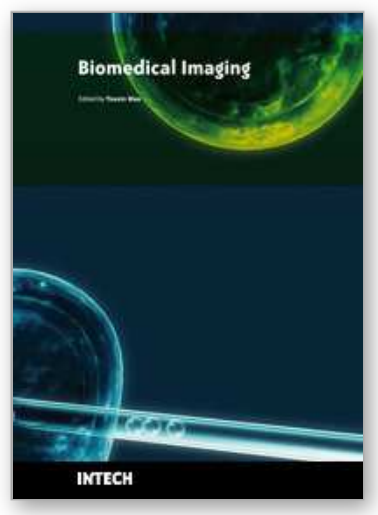

\author{
Biomedical Imaging \\ Edited by Youxin Mao
}

ISBN 978-953-307-071-1

Hard cover, 100 pages

Publisher InTech

Published online 01, March, 2010

Published in print edition March, 2010

Biomedical imaging is becoming an indispensable branch within bioengineering. This research field has recently expanded due to the requirement of high-level medical diagnostics and rapid development of interdisciplinary modern technologies. This book is designed to present the most recent advances in instrumentation, methods, and image processing as well as clinical applications in important areas of biomedical imaging. It provides broad coverage of the field of biomedical imaging, with particular attention to an engineering viewpoint. The goal of the book is to provide a wide-ranging forum in the biomedical imaging field that integrates interdisciplinary research and development of interest to scientists, engineers, teachers, students, and clinical providers.

\title{
How to reference
}

In order to correctly reference this scholarly work, feel free to copy and paste the following:

Youxin Mao, Costel Flueraru and Shoude Chang (2010). Full Range Swept-Source Optical Coherence Tomography with Ultra Small Fiber Probes for Biomedical Imaging, Biomedical Imaging, Youxin Mao (Ed.), ISBN: 978-953-307-071-1, InTech, Available from: http://www.intechopen.com/books/biomedical-imaging/fullrange-swept-source-optical-coherence-tomography-with-ultra-small-fiber-probes-for-biomedical-im

\section{INTECH}

open science | open minds

\section{InTech Europe}

University Campus STeP Ri

Slavka Krautzeka 83/A

51000 Rijeka, Croatia

Phone: +385 (51) 770447

Fax: +385 (51) 686166

www.intechopen.com

\section{InTech China}

Unit 405, Office Block, Hotel Equatorial Shanghai

No.65, Yan An Road (West), Shanghai, 200040, China

中国上海市延安西路65号上海国际贵都大饭店办公楼405单元

Phone: +86-21-62489820

Fax: +86-21-62489821 
(C) 2010 The Author(s). Licensee IntechOpen. This chapter is distributed under the terms of the Creative Commons Attribution-NonCommercialShareAlike-3.0 License, which permits use, distribution and reproduction for non-commercial purposes, provided the original is properly cited and derivative works building on this content are distributed under the same license. 